\title{
Cosmic ray transport in anisotropic magnetohydrodynamic turbulence
}

\section{Mixed magnetosonic and Alfvénic turbulence ${ }^{\star}$}

\author{
A. Shalchi ${ }^{1,2}$ and R. Schlickeiser ${ }^{1}$ \\ ${ }^{1}$ Institut für Theoretische Physik, Lehrstuhl IV: Weltraum- und Astrophysik, Ruhr-Universität Bochum, 44780 Bochum, \\ Germany \\ e-mail: rsch@tp4.ruhr-uni-bochum.de \\ 2 now at: Bartol Research Institute, University of Delaware, Newark, DE 19716, USA
}

Received 10 September 2003 / Accepted 2 February 2004

\begin{abstract}
Observations of interstellar turbulence imply that the power spectrum of the wave turbulence must be highly anisotropic. This anisotropy has to be included when transport of high energy cosmic rays in the Galaxy is discussed. Here we evaluate the relevant cosmic ray transport parameters in the presence of anisotropic plasma wave turbulence, consisting of a mixture of shear Alfvén waves and fast magnetosonic waves. By averaging the respective Fokker-Planck coefficient over the particle pitch-angle we calculate the momentum and spatial diffusion coefficients for different anisotropy parameters. For strongly perpendicular turbulence $(\Lambda \ll 1)$ we obtain that the momentum diffusion coefficient is proportional to $\Lambda^{-1 / 2}\left[\ln \epsilon^{-1}+\ln \Lambda^{1 / 2}\right]$, whereas for strongly parallel turbulence $(\Lambda \gg 1)$ the momentum diffusion coefficient is a constant. We also calculate the anisotropy dependence of the spatial diffusion coefficient and the parallel mean free path for the mixed turbulence. For all coefficients we discuss the rigidity dependence for different cosmic ray particles.
\end{abstract}

Key words. magnetohydrodynamics (MHD) - plasmas - turbulence - ISM: cosmic rays - ISM: magnetic fields acceleration of particles

\section{Introduction}

Observations of interstellar scintillations (Rickett 1990; Spangler 1991), general theoretical considerations (Goldreich $\&$ Sridhar 1995), and comparison of interstellar radiative cooling in HII-regions and in the diffuse interstellar medium with linear Landau damping estimates for fast-mode decay (Lerche \& Schlickeiser 2001a), all strongly imply that the power spectrum of wave turbulence in the interstellar medium must be highly anisotropic. It is not clear from the observations whether the turbulence spectrum is oriented mainly parallel or mainly perpendicular to the ambient magnetic field, either will satisfy the needs of balancing wave damping energy input against radiative cooling. This anisotropy must be included when transport of high energy cosmic rays in the Galaxy is discussed (Chandran 2001; Yan \& Lazarian 2002).

In the first paper of this series (Lerche \& Schlickeiser 2001 b, hereafter referred to LS) we have started to evaluate the relevant cosmic ray transport parameters in the presence of anisotropic fast magnetosonic plasma wave turbulence. All

\footnotetext{
Send offprint requests to: A. Shalchi, e-mail: andreasm4@yahoo.com

* Appendices A, B and C are only available in electronic form at http://www. edpsciences.org
}

technical details of the calculation of Fokker-Planck coefficients and their relation to the transport parameters of the cosmic ray diffusion-convection equation (as the parallel mean free path, the rate of adiabatic deceleration and the momentum diffusion coefficient) were presented there (see LS Sects. 2 and 3). In the second paper of this series (Teufel et al. 2003, hereafter referred to TLS) we continued to evaluate the relevant cosmic ray transport parameters in the presence of anisotropic shear Alfvén waves. However, in order to calculate these transport parameters in the interstellar medium we must calculate also the influence of the anisotropy parameter on the Fokker-Planck coefficients for the mixed turbulence, because interstellar plasma turbulence most probably is a mixture of fast magnetosonic waves and shear Alfvén waves. This analysis is the subject of the present paper. In order to avoid unnecessary repititions we will use the same notation and will frequently refer to equations in papers LS and TLS.

The organisation of the paper is as follows: in Sect. 2 we summarize the results for shear Alfvén waves of TLS. In Sect. 3 we present improved (in comparison to LS) calculations for fast magnetosonic waves. In Sect. 4 we use these new results together with the shear Alfvén wave results to calculate the Fokker-Planck coefficients and transport parameters for 
the mixed turbulence. In Sect. 5 we calculate the rigidity dependence of the transport parameters for heliospheric parameters for shear Alfvén waves, fast magnetosonic waves and the mixed turbulence.

\section{Transport parameters for shear Alfvén waves}

To calculate the transport parameters in the mixed turbulence we need the Fokker-Planck coefficients for shear Alfvén and fast magnetosonic waves. Because the mixed momentum diffusion coefficient $A^{\mathrm{MIX}}$ can be written as the sum of the momentum diffusion coefficients $A^{\mathrm{A}}$ and $A^{\mathrm{F}}$, we write down the Fokker-Planck coefficients and transport parameters of shear Alfvén waves. To do this we can use the results of TLS. For all Fokker-Planck coefficients and transport parameters we have made the assumption that $R_{L} k_{\min } \ll 1$. Therefore we cannot use our results for high rigidities. We also assume that $1<s<2$ for the inertial range spectral index.

\subsection{The momentum diffusion coefficient $A^{\mathrm{A}}$}

For the momentum diffusion coefficient $A^{\mathrm{A}}$ for pure shear Alfvén waves we obtain from TLS(25)

$A^{\mathrm{A}}=\frac{\pi}{2}(s-1) \frac{(\delta B)^{2}}{B_{0}^{2}}\left(k_{\min } R_{L}\right)^{s-1} \frac{v \epsilon^{2} p^{2}}{R_{L}} h^{\mathrm{A}}(\Lambda, s)$.

In Eq. (1) we used the ratio of the turbulent fields and the magnetic background field $\delta B / B_{0}$, the particle speed $v$ and momentum $p$. The parameter $R_{L}$ is the gyroradius and $\epsilon$ is the ratio $v_{\mathrm{A}} / v$ where $v_{\mathrm{A}}$ is the Alfvén speed. The parameter $\Lambda$ is the anisotropy parameter. This parameter allows us to change the turbulence geometry. For $\Lambda=\infty$ we obtain the often considered case of a slab geometry. $\Lambda=1$ is equivalent to isotropic and $\Lambda=0$ is equivalent to 2D geometry (see Bieber et al. 1994; Shalchi \& Schlickeiser 2004). In Eq. (1) we also used the function $h^{\mathrm{A}}$ which can be approximated as follows:

$$
\begin{aligned}
h^{\mathrm{A}}(\Lambda=1, s) \approx & {\left[\left(\frac{1}{2}+\frac{4 \zeta(s+1)}{\pi}\right) \frac{2^{\frac{-s+2}{2}}}{s+2}\right.} \\
& +\left(\frac{1}{s}+\frac{1-2^{\frac{2-s}{2}}}{s(2-s)}\right) \\
& \left.\times\left(\frac{1}{s}-\frac{2^{-s / 2}}{s}+\frac{2^{\frac{-s+2}{2}}}{s+2}-\frac{1}{s+2}\right)\right], \\
h^{\mathrm{A}}(\Lambda \ll 1, s) \approx & {\left[\frac{2 \Lambda^{(s+1) / 2}}{\sqrt{\pi}} \frac{\Gamma\left(\frac{s+2}{2}\right)}{\Gamma\left(\frac{s+1}{2}\right)}\left(\frac{1}{2}+\frac{4 \zeta(s+1)}{\pi}\right)\right.} \\
& \left.\times \frac{1}{2(s+2)}\left(1+\frac{1}{2^{(s+2) / 2}}\right)\right], \\
h^{\mathrm{A}}(\Lambda \gg 1, s) \approx & {\left[\frac{1}{s}-\frac{1}{s+2}\right] . }
\end{aligned}
$$

In Table 1 we compare for the special case $s=5 / 3$ the numerical values of the anisotropy function calculated from these asymptotics with the values calculated from the exact form of
Table 1. Anisotropy function $h^{\mathrm{A}}$ for Alfvén waves and for $s=5 / 3$.

\begin{tabular}{l|l|l}
\hline \hline Anisotropy parameter $\Lambda$ & $h_{\text {ana }}^{\mathrm{A}}(\Lambda, s=5 / 3)$ & $h_{\text {num }}^{\mathrm{A}}(\Lambda, s=5 / 3)$ \\
\hline$\Lambda \ll 1$ & $0.443 \cdot \Lambda^{(s+1) / 2}$ & $0.470 \cdot \Lambda^{(s+1) / 2}$ \\
$\Lambda=1$ & 0.204 & 0.077 \\
$\Lambda \gg 1$ & 0.327 & 0.327 \\
\hline
\end{tabular}

the anisotropy function from TLS. To obtain the results above, we use the Fokker-Planck coefficients of shear Alfvén waves

$$
\begin{aligned}
D_{\mu \mu}^{\mathrm{A}}(\mu \ll \epsilon) \simeq & \frac{\pi(s-1)(\delta B)^{2}\left(R_{L} k_{\min }\right)^{s-1} v}{2 J(\Lambda) B_{0}^{2} R_{L}}|\epsilon|^{s-1} \\
& \times W_{s}^{\mathrm{A}}(\Lambda, \epsilon, s)
\end{aligned}
$$

with

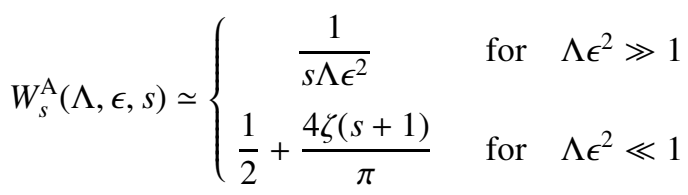

and

$$
\begin{aligned}
D_{\mu \mu}^{\mathrm{A}}(\mu \gg \epsilon) \simeq & \frac{\pi(s-1)(\delta B)^{2}\left(R_{L} k_{\min }\right)^{s-1} v}{2 J(\Lambda) B_{0}^{2} R_{L}}|\mu|^{s+1} \\
& \times W_{l}^{\mathrm{A}}(\Lambda, M, s)
\end{aligned}
$$

with

$$
\begin{aligned}
& W_{l}^{\mathrm{A}}(\Lambda, M, s) \\
& \simeq\left\{\begin{array}{ccc}
\frac{M^{2}}{s \Lambda}\left[1+\frac{1-(1+\Lambda)^{\frac{2-s}{2}}}{(2-s) \Lambda}\right] & \text { for } & M^{2} \ll 1, M^{2} \ll \Lambda \\
\frac{1}{4}+\frac{2 \zeta(s+1)}{\pi} & \text { for } & \Lambda \ll M^{2} \ll 1 \\
\frac{M^{2}}{s \Lambda} & \text { for } & 1 \ll M^{2} \ll \Lambda \\
\frac{1}{2}+\frac{4 \zeta(s+1)}{\pi} & \text { for } & M^{2} \gg 1, M^{2} \gg \Lambda
\end{array}\right.
\end{aligned}
$$

where

$M=\frac{\sqrt{1-\mu^{2}}}{\mu}$.

For the function $J(\Lambda, s)$ we have

$$
\begin{aligned}
J(\Lambda, s) & \approx{ }_{2} F_{1}\left(\frac{s+2}{2}, 1 ; \frac{3}{2} ; 1-\Lambda\right) \\
& \simeq\left\{\begin{array}{ccc}
\frac{\sqrt{\pi}}{2 \Lambda^{(s+1) / 2} \frac{\Gamma\left(\frac{s+1}{2}\right)}{\Gamma\left(\frac{s+2}{2}\right)}} & \text { for } & \Lambda \ll 1 \\
1 & \text { for } & \Lambda=1 \\
\frac{1}{s \Lambda} & \text { for } & \Lambda \gg 1 .
\end{array}\right.
\end{aligned}
$$


Table 2. The function $g^{\mathrm{A}}$ for Alfvén waves and for $s=5 / 3$.

\begin{tabular}{l|l}
\hline \hline Anisotropy parameter $\Lambda$ & $g^{\mathrm{A}}(\Lambda, \epsilon, s=5 / 3)$ \\
\hline$\Lambda \ll 1$ & $0.63 \epsilon^{-s} \Lambda^{-(s+1) / 2}$ \\
$\Lambda=1$ & $0.75 \epsilon^{-s}$ \\
$\epsilon^{-s} \gg \Lambda \gg 1$ & $0.45 \epsilon^{-s} \Lambda^{-1}$ \\
$\Lambda \gg \epsilon^{-s} \gg 1$ & 2.57 \\
\hline
\end{tabular}

\subsection{The spatial diffusion coefficient $\kappa^{\mathrm{A}}$}

Here we use the results from TLS again to obtain

$\kappa^{\mathrm{A}}=\frac{v B_{0}^{2} R_{L}^{2-s}}{2 \pi(s-1) \delta B^{2} k_{\min }^{s-1}} g^{\mathrm{A}}(s, \epsilon, \Lambda)$

with the function

$g^{\mathrm{A}}\left(s, \epsilon^{-s} \gg \Lambda\right)=J(\Lambda, s) \cdot \frac{1}{\epsilon^{s}} \frac{2 \pi(s+1)}{s(\pi+8 \zeta(s+1))}$

if $\epsilon^{-s} \gg \Lambda$, and

$g^{\mathrm{A}}\left(s, \Lambda \gg \epsilon^{-s} \gg 1\right)=J(\Lambda, s) \cdot \frac{2}{(2-s)(4-s)}$

if $\Lambda \gg \epsilon^{-s} \gg 1$. In Table 2 we show the values of the function $g^{\mathrm{A}}$ for the special case $s=5 / 3$.

\section{Transport parameters for fast magnetosonic waves}

\subsection{The momentum diffusion coefficient $A^{\mathrm{F}}$}

To calculate the transport parameters for the mixed turbulence we also need the parameters for fast magnetosonic waves. For these waves the momentum diffusion coefficient is the sum of the gyroresonance contribution $A^{\mathrm{G}}$ and the transit-time damping contribution $A^{\mathrm{T}}$,

$A^{\mathrm{F}}=A^{\mathrm{G}}+A^{\mathrm{T}}$

because we know that

$D_{\mu \mu}^{\mathrm{F}}=D_{\mu \mu}^{\mathrm{G}}+D_{\mu \mu}^{\mathrm{T}}$.

\subsubsection{Transit-time damping}

To calculate $A^{\mathrm{T}}$ we use the results from LS that

$$
\begin{aligned}
D_{\mu \mu}^{\mathrm{T}}(\mu \gg \epsilon)= & \frac{\pi(s-1) v R_{L}^{s-2} k_{\min }^{s-1}}{2 J(\Lambda)} \frac{\delta B^{2}}{B_{0}^{2}} \\
& \times c_{1}(\mu, R, s) F(\mu, \epsilon, \Lambda, s)
\end{aligned}
$$

and

$D_{\mu \mu}^{\mathrm{T}}(\mu \leq \epsilon)=0$,

respectively, where we introduce $R=R_{L} k_{\min }$ and define the two functions

$c_{1}(\mu, R, s)=\int_{R}^{\infty} \sqrt{1-\mu^{2}} d x x^{-(s+1)} J_{1}^{2}(x)$
Table 3. Anisotropy function $h^{\mathrm{T}}$ for fast magnetosonic waves. In this table we introduced the functions $\xi(s)=\frac{c_{1}(s) \Gamma(1+s / 2)}{\sqrt{\pi} \Gamma((s+1) / 2)} B\left(\frac{s+4}{2}, \frac{s+2}{2}\right)$ with the generalized $\beta$-function $B(x, y)$ and $c_{3}(s)=\frac{\Gamma(1-s / 2)}{\pi \Gamma(2+s / 2)}$.

\begin{tabular}{l|l}
\hline \hline Anisotropy parameter $\Lambda$ & $h^{\mathrm{T}}(\Lambda, \epsilon, s)$ \\
\hline$\Lambda \ll \epsilon^{2} \ll 1$ & $\xi(s) \Lambda^{\frac{s+1}{2}} \epsilon^{-(s+2)}$ \\
$\epsilon^{2} \ll \Lambda \ll 1$ & $c_{3}(s) \Lambda^{-1 / 2}\left[\ln \epsilon^{-1}+\ln \Lambda^{1 / 2}\right]$ \\
$\Lambda=1$ & $c_{1}(s) \ln \epsilon^{-1}$ \\
$\Lambda \gg 1$ & $c_{1}(s) s \Lambda^{-s / 2} \ln \epsilon^{-1}$ \\
\hline
\end{tabular}

and

$$
\begin{aligned}
F(\mu, \epsilon, \Lambda, s) \approx & \frac{\left(1-\mu^{2}\right)^{(s+2) / 2} \mu^{s+1}}{\left[\epsilon^{2}+\Lambda\left(\mu^{2}-\epsilon^{2}\right)\right]^{(s+2) / 2}} \\
& \approx\left\{\begin{array}{cl}
\frac{\left(1-\mu^{2}\right)^{(s+2) / 2} \mu^{s+1}}{\epsilon^{s+2}} & \text { for } \Lambda \ll 1, \mu \ll \frac{\epsilon}{\sqrt{\Lambda}} \\
\frac{\left(1-\mu^{2}\right)^{(s+2) / 2}}{\mu \Lambda^{(s+2) / 2}} & \text { for } \Lambda \ll 1, \mu \gg \frac{\epsilon}{\sqrt{\Lambda}} \\
\frac{\left(1-\mu^{2}\right)^{(s+2) / 2}}{\mu} & \text { for } \quad \Lambda=1 \\
\frac{\left(1-\mu^{2}\right)^{(s+2) / 2}}{\mu \Lambda^{(s+2) / 2}} & \text { for } \quad \Lambda \gg 1 .
\end{array}\right.
\end{aligned}
$$

In this paper we restrict our analysis to $R_{L} k_{\min } \ll 1$. Then the approximation

$$
\begin{aligned}
c_{1}(\mu, R, s) & \approx \int_{0}^{\infty} \mathrm{d} x x^{-(s+1)} J_{1}^{2}(x) \\
& =c_{1}(s)=\frac{2^{1-s} s}{4-s^{2}} \frac{\Gamma(s) \Gamma\left(2-\frac{s}{2}\right)}{\Gamma^{3}\left(1+\frac{s}{2}\right)}
\end{aligned}
$$

holds, and we obtain for the momentum diffusion coefficient of the transit-time damping

$A^{\mathrm{T}}=\frac{\pi}{2}(s-1) \frac{(\delta B)^{2}}{B_{0}^{2}}\left(k_{\min } R_{L}\right)^{s-1} \frac{v \epsilon^{2} p^{2}}{R_{L}} h^{\mathrm{T}}(\Lambda, \epsilon, s)$

with the anisotropy function $h^{\mathrm{T}}$ listed in Table 3.

\subsubsection{Gyroresonance contribution}

The Fokker-Planck coefficient for the gyroresonance contribution has also been calculated in LS, but it is possible to derive improved approximations using Kapteyn series, as is shown in Appendix C. For small pitch angles $\mu \ll \epsilon$ and for $R_{L} k_{\min } \ll 1$ we obtain from Appendix B

$$
\begin{aligned}
D_{\mu \mu}^{\mathrm{G}}(\mu \ll \epsilon) \approx & \frac{\pi(s-1)(\delta B)^{2}\left(R_{L} k_{\min }\right)^{s-1} v}{2 J(\Lambda) B_{0}^{2} R_{L}}|\epsilon|^{s-1} \\
& \times W_{s}^{\mathrm{G}}(\Lambda, \epsilon, s)
\end{aligned}
$$


with

$$
W_{s}^{\mathrm{G}}(\Lambda, \epsilon, s) \simeq\left\{\begin{array}{ccc}
\frac{c_{2} \epsilon}{2 \Lambda^{(s+1) / 2}} & \text { for } & \Lambda \ll 1 \\
\frac{3 \zeta(s+1) \epsilon}{4} & \text { for } & \Lambda=1 \\
\frac{c_{2} \epsilon}{\sqrt{\Lambda}} & \text { for } & \epsilon^{-2} \gg \Lambda \gg 1 \\
\frac{1}{2 s \Lambda} & \text { for } & \Lambda \gg \epsilon^{-2} \gg 1
\end{array}\right.
$$

where we introduced

$c_{2}=c_{2}(s)=\frac{2 \zeta(s+1) \Gamma\left(\frac{s+3}{2}\right)}{\sqrt{\pi}(s+1) \Gamma\left(\frac{s+2}{2}\right)}=\frac{\zeta(s+1)}{\sqrt{\pi}} \frac{\Gamma\left(\frac{s+1}{2}\right)}{\Gamma\left(\frac{s+2}{2}\right)}$.

For large pitch angles $\mu \gg \epsilon$ and $R_{L} k_{\min } \ll 1$ we obtain from Appendix A

$$
\begin{aligned}
D_{\mu \mu}^{\mathrm{G}}(\mu \gg \epsilon) \approx & \frac{\pi(s-1)(\delta B)^{2}\left(R_{L} k_{\min }\right)^{s-1} v}{J(\Lambda) B_{0}^{2} R_{L}}|\mu|^{s-1}\left(1-\mu^{2}\right) \\
& \times W_{l}^{\mathrm{G}}(\Lambda, M, s)
\end{aligned}
$$

with

$$
W_{l}^{\mathrm{G}}(\Lambda, M, s) \simeq\left\{\begin{array}{ccc}
\frac{1}{s \Lambda} & \text { for } & M^{2} \ll \Lambda \\
\frac{c_{2}}{M \sqrt{\Lambda}} & \text { for } & \Lambda \ll M^{2} \ll 1 \\
\frac{2 c_{2}}{M \sqrt{\Lambda}} & \text { for } & \Lambda \ll M^{2}, M^{2} \gg 1
\end{array}\right.
$$

and with

$M=\frac{\sqrt{1-\mu^{2}}}{\mu}$.

With the definition of the momentum diffusion coefficient $A$ (Eq. LS(9)) we find for cosmic rays with gyroradii much less than $R_{L} k_{\min } \ll 1$

$A^{\mathrm{G}}=\frac{\pi}{2}(s-1) \frac{(\delta B)^{2}}{B_{0}^{2}}\left(k_{\min } R_{L}\right)^{s-1} \frac{v \epsilon^{2} p^{2}}{R_{L}} h^{\mathrm{G}}(\Lambda, \epsilon, s)$

with the anisotropy function

$$
\begin{aligned}
h^{\mathrm{G}}(\Lambda, \epsilon, s)= & \frac{1}{J(\Lambda)} \int_{0}^{\epsilon} \mathrm{d} \mu \epsilon^{s-1} W_{s}^{\mathrm{G}}(\mu) \\
& +\frac{1}{J(\Lambda)} \int_{\epsilon}^{1} \mathrm{~d} \mu \mu^{s-1}\left(1-\mu^{2}\right) W_{l}^{\mathrm{G}}(\mu) .
\end{aligned}
$$

This anisotropy function can again be simplified for different limits of the anisotropy parameter $\Lambda$. We discuss the different limits in turn.

\subsubsection{Isotropic turbulence $\Lambda=1$}

With the results of Appendix A it is easy to determine $W_{l}^{\mathrm{G}}$ in this case:

$$
W_{l}^{\mathrm{G}}=\left\{\begin{array}{ccc}
\frac{1}{s} & \text { for } & M^{2} \ll 1 \\
\frac{2 c_{2}}{M} & \text { for } & M^{2} \gg 1
\end{array}\right.
$$

For $h^{\mathrm{G}}$ we use $J(\Lambda=1, s)=1$ and split the integral as

$$
\begin{aligned}
h^{\mathrm{G}}(\Lambda=1, \epsilon, s) \approx & \frac{3 \zeta(s+1)}{4} \epsilon^{s} \int_{0}^{\epsilon} \mathrm{d} \mu \\
& +2 c_{2} \int_{\epsilon}^{1 / \sqrt{2}} \mathrm{~d} \mu \mu^{s} \sqrt{1-\mu^{2}} \\
& +\frac{1}{s} \int_{1 / \sqrt{2}}^{1} \mathrm{~d} \mu\left(\mu^{s-1}-\mu^{s+1}\right) .
\end{aligned}
$$

These integrals are elementary and we obtain to lowest order in $\epsilon$

$$
\begin{aligned}
h^{\mathrm{G}}(\Lambda=1, s) \approx & \frac{c_{2}}{(s+1) 2^{(s-1) / 2}}{ }_{2} F_{1}\left(-\frac{1}{2}, \frac{s+1}{2} ; \frac{s+3}{2} ; \frac{1}{2}\right) \\
& +\frac{1}{s}\left[\frac{1}{s}-\frac{1}{s+2}-\frac{1}{s 2^{s / 2}}+\frac{1}{(s+2) 2^{(s+2) / 2}}\right] .
\end{aligned}
$$

\subsubsection{Strongly perpendicular turbulence $\epsilon^{\frac{2(s+1)}{s}} \ll \Lambda \ll 1$}

In this case we obtain from Appendix A:

$W_{l}^{\mathrm{G}}=\left\{\begin{array}{lll}\frac{1}{s \Lambda} & \text { for } & M^{2} \ll \Lambda \ll 1 \\ \frac{c_{2}}{M \sqrt{\Lambda}} & \text { for } & \Lambda \ll M^{2} \ll 1 \\ \frac{2 c_{2}}{M \sqrt{\Lambda}} & \text { for } & \Lambda \ll 1 \ll M^{2} .\end{array}\right.$

For $h^{\mathrm{G}}$ we now find

$$
\begin{aligned}
h^{\mathrm{G}}\left(\epsilon^{\frac{2(s+1)}{s}} \ll \Lambda \ll 1, s\right) & \approx \frac{1}{J(\Lambda)}\left\{\frac{c_{2} \epsilon^{s+1}}{2 \Lambda^{(s+1) / 2}}\right. \\
& +\frac{2 c_{2}}{\sqrt{\Lambda}} \int_{\epsilon}^{1 / \sqrt{2}} \mathrm{~d} \mu \mu^{s} \sqrt{1-\mu^{2}} \\
& +\frac{c_{2}}{\sqrt{\Lambda}} \int_{1 / \sqrt{2}}^{1 / \sqrt{1+\Lambda}} \mathrm{d} \mu \mu^{s} \sqrt{1-\mu^{2}} \\
& \left.+\frac{1}{s \Lambda} \int_{1 / \sqrt{1+\Lambda}}^{1} \mathrm{~d} \mu\left(\mu^{s-1}-\mu^{s+1}\right)\right\} .
\end{aligned}
$$

Again these integrals are elementary, so that

$$
h^{\mathrm{G}}\left(\epsilon^{2(s+1) / s} \ll \Lambda \ll 1, s\right)=\frac{1}{J(\Lambda)} \frac{c_{2} \sqrt{\pi} \Gamma\left(\frac{s+3}{2}\right)}{2(s+1) \Gamma\left(\frac{s+4}{2}\right)} \frac{1}{\sqrt{\Lambda}}
$$

to lowest order in $\epsilon$. With

$$
J(\Lambda \ll 1, s) \approx \frac{1}{\Lambda^{\frac{s+1}{2}}}{ }_{2} F_{1}\left(\frac{1-s}{2}, \frac{1}{2}, \frac{3}{2}, 1\right)=\frac{\sqrt{\pi}}{2 \Lambda^{\frac{s+1}{2}}} \frac{\Gamma\left(\frac{s+1}{2}\right)}{\Gamma\left(\frac{s+2}{2}\right)}
$$

we find for the anisotropy function $h^{\mathrm{G}}$

$h^{\mathrm{G}}\left(\epsilon^{2(s+1) / s} \ll \Lambda \ll 1, s\right) \approx \frac{c_{2}}{s+2} \Lambda^{s / 2}$. 


\subsubsection{Extremely perpendicular turbulence}

$$
\Lambda \ll \epsilon^{\frac{2(s+1)}{s}} \ll 1
$$

The calculations are similar as before. Here we find:

$h^{\mathrm{G}}\left(\Lambda \ll \epsilon^{2 / s(s+1)} \ll 1, s\right) \approx \frac{1}{J(\Lambda, s)} \frac{c_{2}(s)}{2} \frac{\epsilon^{s+1}}{\Lambda^{(s+1) / 2}}$

and therefore

$h^{\mathrm{G}}\left(\Lambda \ll \epsilon^{2 / s(s+1)} \ll 1, s\right) \approx \frac{c_{2}(s) \Gamma\left(\frac{s+2}{2}\right)}{\sqrt{\pi} \Gamma\left(\frac{s+1}{2}\right)} \epsilon^{s+1}$.

Because $\epsilon \ll 1$ this implies very small values of $h^{\mathrm{G}} \ll 1$.

\subsubsection{Strongly parallel turbulence $\epsilon^{-2} \gg \Lambda \gg 1$}

In this case we obtain:

$W_{l}^{\mathrm{G}}=\left\{\begin{array}{ccc}\frac{1}{s \Lambda} & \text { for } & M^{2} \ll \Lambda \\ \frac{2 c_{2}}{M \sqrt{\Lambda}} & \text { for } & M^{2} \gg \Lambda .\end{array}\right.$

For $h^{\mathrm{G}}$ we now find to lowest order in $\epsilon$

$$
\begin{aligned}
h^{\mathrm{G}}(\Lambda \gg 1, s) \approx & \frac{1}{J(\Lambda)}\left\{\frac{c_{2} \epsilon^{s+1}}{\sqrt{\Lambda}}+\frac{2 c_{2}}{\sqrt{\Lambda}} \int_{\epsilon}^{1 / \sqrt{1+\Lambda}} \mathrm{d} \mu \mu^{s} \sqrt{1-\mu^{2}}\right. \\
& \left.+\frac{1}{s \Lambda} \int_{1 / \sqrt{1+\Lambda}}^{1} \mathrm{~d} \mu\left(\mu^{s-1}-\mu^{s+1}\right)\right\}
\end{aligned}
$$

Like in the cases before the integrals are elementary and we obtain

$h^{\mathrm{G}}\left(\epsilon^{-2} \gg \Lambda \gg 1, s\right) \approx \frac{1}{J(\Lambda)} \frac{1}{s \Lambda}\left[\frac{1}{s}-\frac{1}{s+2}\right]$.

With

$J(\Lambda \gg 1, s) \approx \frac{1}{s \Lambda}$

we finally find

$h^{\mathrm{G}}\left(\epsilon^{-2} \gg \Lambda \gg 1, s\right)=\frac{1}{s}-\frac{1}{s+2}$.

\subsubsection{Extremely parallel turbulence $\Lambda \gg \epsilon^{-2} \gg 1$}

In this case we have:

$W_{l}^{\mathrm{G}}=\left\{\begin{array}{ccc}\frac{1}{s \Lambda} & \text { for } & M^{2} \ll \Lambda \\ \frac{2 c_{2}}{M \sqrt{\Lambda}} & \text { for } & M^{2} \gg \Lambda .\end{array}\right.$

For $h^{\mathrm{G}}$ we then find

$$
\begin{aligned}
h^{\mathrm{G}}\left(\Lambda \gg \epsilon^{-2} \gg 1, s\right) \approx & \frac{1}{J(\Lambda)}\left\{\frac{\epsilon^{s}}{2 s \Lambda}\right. \\
& +\frac{2 c_{2}}{\sqrt{\Lambda}} \int_{\epsilon}^{1 / \sqrt{1+\Lambda}} \mathrm{d} \mu \mu^{s} \sqrt{1-\mu^{2}} \\
& \left.+\frac{1}{s \Lambda} \int_{1 / \sqrt{1+\Lambda}}^{1} \mathrm{~d} \mu\left(\mu^{s-1}-\mu^{s+1}\right)\right\}
\end{aligned}
$$

Table 4. Anisotropy function $h^{\mathrm{G}}$ for fast magnetosonic waves.

\begin{tabular}{l|l|l}
\hline \hline Anisotropy parameter $\Lambda$ & $h_{\mathrm{num}}^{\mathrm{G}}(\Lambda, s=5 / 3)$ & $h_{\mathrm{ana}}^{\mathrm{G}}(\Lambda, s=5 / 3)$ \\
\hline$\epsilon^{2(s+1) / s} \ll \Lambda \ll 1$ & $0.178 \Lambda^{s / 2}$ & $0.188 \Lambda^{s / 2}$ \\
$\Lambda=1$ & 0.067 & 0.212 \\
$\Lambda \gg 1$ & 0.326 & 0.327 \\
\hline
\end{tabular}

Table 5. Anisotropy function $h^{\mathrm{F}}$ for fast magnetosonic waves.

\begin{tabular}{l|l}
\hline \hline Anisotropy parameter $\Lambda$ & $h^{\mathrm{F}}(\Lambda, \epsilon, s)$ \\
\hline$\epsilon^{2} \ll \Lambda \ll 1$ & $c_{3}(s) \Lambda^{-1 / 2}\left[\ln \epsilon^{-1}+\ln \Lambda^{1 / 2}\right]$ \\
$\Lambda=1$ & $c_{1}(s) \ln \epsilon^{-1}$ \\
$\Lambda \gg 1$ & $\frac{1}{s}-\frac{1}{s+2}$ \\
\hline
\end{tabular}

and to lowest order in $\epsilon$ we find the same result as in the case before.

$h^{\mathrm{G}}\left(\Lambda \gg \epsilon^{-2} \gg 1, s\right)=\frac{1}{s}-\frac{1}{s+2}$.

The combined result for the case $\Lambda \gg 1$ therefore is

$h^{\mathrm{G}}(\Lambda \gg 1, s) \approx \frac{1}{s}-\frac{1}{s+2}$.

In Table 4 we list the results for the different cases of anisotropy for the special turbulence spectral index value $s=5 / 3$. We also calculate the three cases numerically from the exact expression. As can be seen, the agreement is very good in cases one and three and acceptable in the second case.

Knowing the variation of the two anisotropy functions from transit-time damping $\left(h^{\mathrm{T}}\right)$ and gyroresonant interactions $\left(h^{\mathrm{G}}\right)$, we are now able to calculate $A^{\mathrm{F}}$ :

$A^{\mathrm{F}}=\frac{\pi}{2}(s-1) \frac{(\delta B)^{2}}{B_{0}^{2}}\left(k_{\min } R_{L}\right)^{s-1} \frac{v \epsilon^{2} p^{2}}{R_{L}} h^{\mathrm{F}}(\Lambda, \epsilon, s)$

with

$h^{\mathrm{F}}(\Lambda, \epsilon, s)=h^{\mathrm{T}}(\Lambda, \epsilon, s)+h^{\mathrm{G}}(\Lambda, s)$.

The behaviour of the function $h^{\mathrm{F}}(\Lambda, \epsilon, s)$ is summarized in Table 5: for the cases $\epsilon^{2} \ll \Lambda \ll 1$ and $\Lambda=1$ the momentum diffusion coefficient $A^{\mathrm{F}}$ can be approximated solely by the transit-time damping contribution $A^{\mathrm{T}}$, whereas for the case $\Lambda \gg 1$ (parallel turbulence) the gyroresonance contribution dominates the sum, because of the lack of perpendicular waves which are essential for transit-time damping.

\subsection{The spatial diffusion coefficient $\kappa^{\mathrm{F}}$}

We restrict our analysis to $\epsilon^{2} \ll \Lambda$. From LS we know that

$D_{\mu \mu}^{\mathrm{T}}(\mu \leq \epsilon)=0$.

With the function

$D_{\mu \mu}^{\mathrm{G}}(\mu \ll \epsilon)=\frac{\pi(s-1) R_{L}^{s-2} v \epsilon^{s-1} k_{\min }^{s-1}}{2 J(\Lambda)} \frac{(\delta B)^{2}}{B_{0}^{2}} W_{s}^{\mathrm{G}}(\Lambda, \epsilon, s)$ 
from Appendix B we obtain for the spatial diffusion coefficient

$$
\begin{aligned}
\kappa^{\mathrm{F}}= & \frac{v^{2}}{4} \int_{0}^{1} \mathrm{~d} \mu \frac{\left(1-\mu^{2}\right)^{2}}{D_{\mu \mu}^{\mathrm{F}}(\mu)} \\
\approx & \frac{v^{2}}{4} \int_{0}^{\epsilon} \frac{\mathrm{d} \mu}{D_{\mu \mu}^{\mathrm{G}}(\mu \ll \epsilon)} \\
& +\frac{v^{2}}{4} \int_{\epsilon}^{1} \mathrm{~d} \mu \frac{\left(1-\mu^{2}\right)^{2}}{D_{\mu \mu}^{\mathrm{G}}(\mu \gg \epsilon)+D_{\mu \mu}^{\mathrm{T}}(\mu \gg \epsilon)}
\end{aligned}
$$

which can be written as

$\kappa^{\mathrm{F}}=\frac{v R_{L}^{2-s}}{2 \pi(s-1) k_{\min }^{s-1}} \frac{B_{0}^{2}}{\delta B^{2}} g^{\mathrm{F}}(\Lambda, \epsilon, s)$

where we introduced the function

$$
\begin{aligned}
g^{\mathrm{F}}(\Lambda, \epsilon, s)= & \frac{J(\Lambda, s) \epsilon^{1-s}}{W_{s}(\Lambda, \epsilon, s)} \int_{0}^{\epsilon} \mathrm{d} \mu \\
& +J(\Lambda, s) \int_{\epsilon}^{1} \mathrm{~d} \mu \frac{\left(1-\mu^{2}\right)^{2}}{\mu^{s-1}\left(1-\mu^{2}\right) W_{l}+c_{1} F(\mu)}
\end{aligned}
$$

with the functions $W_{s}^{\mathrm{G}}, W_{l}^{\mathrm{G}}$ and $F(\mu, \epsilon, \Lambda, s)$ defined in Eqs. (21), (24) and (17), respectively. These integrals can be solved analytically for special cases of $\Lambda$ again.

\subsubsection{Isotropic turbulence $\wedge=1$}

Here we find

$$
\begin{aligned}
& g^{\mathrm{F}}(\Lambda=1, \epsilon, s) \approx \int_{0}^{\epsilon} \mathrm{d} \mu \frac{4 \epsilon^{-s}}{3 \zeta(s+1)} \\
& \quad+\int_{\epsilon}^{1 / \sqrt{2}} \mathrm{~d} \mu \frac{\left(1-\mu^{2}\right)^{2}}{2 c_{2} \mu^{s-1}\left(1-\mu^{2}\right)^{2} M^{-1}+c_{1}\left(1-\mu^{2}\right)^{(s+2) / 2} \mu^{-1}} \\
& \quad+\int_{1 / \sqrt{2}}^{1} \mathrm{~d} \mu \frac{\left(1-\mu^{2}\right)^{2}}{s^{-1} \mu^{s-1}\left(1-\mu^{2}\right)^{2}+c_{1}\left(1-\mu^{2}\right)^{(s+2) / 2} \mu^{-1}} .
\end{aligned}
$$

where we have used $J(\Lambda=1)=1$. It is easy to see that the second and third integral are of higher order in $\epsilon \ll 1$, so that the function $g^{\mathrm{F}}$ is determined by the first integral:

$g^{\mathrm{F}}(\Lambda=1, \epsilon, s) \approx \frac{4 \epsilon^{1-s}}{3 \zeta(s+1)}$.

\subsubsection{Strongly perpendicular turbulence $\epsilon^{2} \ll \wedge \ll 1$}

For this case we find

$$
\begin{aligned}
& g^{\mathrm{F}}(\Lambda=1, \epsilon, s) \approx J(\Lambda, s)\left\{\int_{0}^{\epsilon} \mathrm{d} \mu \frac{2 \Lambda^{(s+1) / 2}}{c_{2} \epsilon^{s}}\right. \\
& +\int_{\epsilon}^{\epsilon / \sqrt{\Lambda}} \mathrm{d} \mu \frac{1}{\frac{2 c_{2}}{M \Lambda^{1 / 2}} \mu^{s-1}+c_{1} \mu^{s+1} \epsilon^{-2-s}} \\
& +\int_{\epsilon / \sqrt{\Lambda}}^{1 / \sqrt{2}} \mathrm{~d} \mu \frac{\left(1-\mu^{2}\right)^{2}}{\frac{2 c_{2}}{M \Lambda^{1 / 2}}\left(1-\mu^{2}\right) \mu^{s-1}+\frac{c_{1}}{\mu}\left(1-\mu^{2}\right)^{(s+2) / 2} \Lambda^{-\frac{(2+s)}{2}}} \\
& +\int_{1 / \sqrt{2}}^{1 / \sqrt{1+\Lambda}} \mathrm{d} \mu \frac{\left(1-\mu^{2}\right)^{2}}{\frac{c_{2}}{M \Lambda^{1 / 2}}\left(1-\mu^{2}\right) \mu^{s-1}+\frac{c_{1}}{\mu}\left(1-\mu^{2}\right)^{(s+2) / 2} \Lambda^{-\frac{(2+s)}{2}}} \\
& \left.+\int_{1 / \sqrt{1+\Lambda}}^{1} \mathrm{~d} \mu \frac{\left(1-\mu^{2}\right)^{2}}{\frac{1}{s \Lambda}\left(1-\mu^{2}\right) \mu^{s-1}+\frac{c_{1}}{\mu}\left(1-\mu^{2}\right)^{(s+2) / 2} \Lambda^{-\frac{(2+s)}{2}}}\right\}
\end{aligned}
$$

With $M=\sqrt{1-\mu^{2}} / \mu$ this can be written as

$$
\begin{aligned}
& g^{\mathrm{F}}(\Lambda=1, \epsilon, s) \approx J(\Lambda, s)\left\{\frac{2 \Lambda^{(s+1) / 2}}{c_{2} \epsilon^{s-1}}+\int_{\epsilon}^{\epsilon / \sqrt{\Lambda}} \mathrm{d} \mu \frac{\epsilon^{s+2}}{c_{1} \mu^{s+1}}\right. \\
& \quad+\int_{\epsilon / \sqrt{\Lambda}}^{1 / \sqrt{2}} \mathrm{~d} \mu \frac{\left(1-\mu^{2}\right)^{2}}{\mu^{s+1}\left[2 c_{2} M \Lambda^{-1 / 2}+c_{1} M^{s+2} \Lambda^{-(2+s) / 2}\right]} \\
& +\int_{1 / \sqrt{2}}^{1 / \sqrt{1+\Lambda}} \mathrm{d} \mu \frac{\left(1-\mu^{2}\right)^{2}}{\mu^{s+1}\left[c_{2} M \Lambda^{-1 / 2}+c_{1} M^{s+2} \Lambda^{-(2+s) / 2}\right]} \\
& \left.+\int_{1 / \sqrt{1+\Lambda}}^{1} \mathrm{~d} \mu \frac{\left(1-\mu^{2}\right)^{2}}{\mu^{s+1}\left[s^{-1} M^{2} \Lambda^{-1}+c_{1} M^{s+2} \Lambda^{-(2+s) / 2}\right]}\right\}
\end{aligned}
$$

Now we use $M \Lambda^{-1 / 2} \gg 1$ in the second and third integral and $M \Lambda^{-1 / 2} \ll 1$ in the last integral. To lowest order in $\epsilon$ and for $\Lambda \ll 1$ we find that the first term dominates

$g^{\mathrm{F}}(\Lambda \ll 1, \epsilon, s) \approx J(\Lambda) \frac{2 \Lambda^{(s+1) / 2}}{c_{2} \epsilon^{s-1}}$

With

$J(\Lambda \ll 1) \approx \frac{\pi c_{2}}{2 \zeta(s+1)} \Lambda^{-(s+1) / 2}$

we then obtain

$g^{\mathrm{F}}(\Lambda \ll 1, \epsilon, s) \approx \frac{\pi}{\zeta(s+1) \epsilon^{s-1}}$.

\subsubsection{Strongly parallel turbulence $\epsilon^{-2} \gg \wedge \gg 1$}

Here we obtain

$$
\begin{aligned}
& g^{\mathrm{F}}(\Lambda \gg 1, \epsilon, s) \approx J(\Lambda, s)\left\{\int_{0}^{\epsilon} \mathrm{d} \mu \frac{\sqrt{\Lambda}}{c_{2} \epsilon^{s}}\right. \\
& \quad+\int_{\epsilon}^{1 / \sqrt{1+\Lambda}} \mathrm{d} \mu \frac{1}{2 c_{2} M^{-1} \Lambda^{-1 / 2} \mu^{s-1}+c_{1} \mu^{s} \Lambda^{-\frac{(s+2)}{2}}} \\
& \left.\quad+\int_{1 / \sqrt{1+\Lambda}}^{1} \mathrm{~d} \mu \frac{\left(1-\mu^{2}\right)^{2}}{\frac{1}{s \Lambda} \mu^{s-1}\left(1-\mu^{2}\right)+\frac{c_{1}}{\mu}\left(1-\mu^{2}\right)^{\frac{(s+2)}{2}} \Lambda^{-\frac{(s+2)}{2}}}\right\} .
\end{aligned}
$$

With $M=\sqrt{1-\mu^{2}} / \mu$ this can be written as

$$
\begin{aligned}
& g^{\mathrm{F}}(\Lambda \gg 1, \epsilon, s) \approx J(\Lambda, s)\left\{\frac{\sqrt{\Lambda}}{c_{2} \epsilon^{s-1}}\right. \\
& \quad+\int_{\epsilon}^{1 / \sqrt{1+\Lambda}} \mathrm{d} \mu \frac{1}{\mu^{s+1}\left[2 c_{2} M \Lambda^{-1 / 2}+c_{1} M^{s+2} \Lambda^{-(s+2) / 2}\right]} \\
& \left.\quad+\int_{1 / \sqrt{1+\Lambda}}^{1} \mathrm{~d} \mu \frac{\left(1-\mu^{2}\right)^{2}}{\mu^{s+1}\left[s^{-1} M^{2} \Lambda^{-1}+c_{1} M^{s+2} \Lambda^{-(s+2) / 2}\right]}\right\} .
\end{aligned}
$$

We use $M \Lambda^{-1 / 2} \gg 1$ in the first integral and $M \Lambda^{-1 / 2} \ll 1$ in the second integral. To lowest order in $\epsilon$ and for $\Lambda \gg 1$ we find

$g^{\mathrm{F}}(\Lambda=1, \epsilon, s) \approx \frac{1}{s c_{2} \sqrt{\Lambda} \cdot \epsilon^{s-1}}$. 
Table 6. Anisotropy function $g^{\mathrm{F}}$ for fast magnetosonic turbulence.

\begin{tabular}{l|l|l}
\hline \hline Anisotropy parameter $\Lambda$ & $g^{\mathrm{F}}(\Lambda, \epsilon, s)$ & $g^{\mathrm{F}}(\Lambda, \epsilon, s=5 / 3)$ \\
\hline$\epsilon^{2} \ll \Lambda \ll 1$ & $\frac{\pi}{\zeta(s+1)} \epsilon^{1-s}$ & $2.446 \epsilon^{1-s}$ \\
$\Lambda=1$ & $\frac{4}{3 \zeta(s+1)} \epsilon^{1-s}$ & $1.038 \epsilon^{1-s}$ \\
$\epsilon^{-2} \gg \Lambda \gg 1$ & $\frac{1}{s c_{2}} \epsilon^{1-s} \Lambda^{-1 / 2}$ & $0.872 \epsilon^{1-s} \Lambda^{-1 / 2}$ \\
$\Lambda \gg \epsilon^{-2} \gg 1$ & $\frac{2}{(2-s)(4-s)}$ & 2.57 \\
\hline
\end{tabular}

\subsection{Extremely parallel turbulence $\Lambda \gg \epsilon^{-2} \gg 1$}

For this case we find

$$
\begin{aligned}
& g^{\mathrm{F}}\left(\Lambda \gg \epsilon^{-2} \gg 1, s\right) \approx 2 \epsilon^{2-s} \\
& +\frac{1}{s \Lambda} \int_{\epsilon}^{1 / \sqrt{1+\Lambda}} \mathrm{d} \mu \frac{\left(1-\mu^{2}\right)^{2}}{\mu^{s-1}\left(1-\mu^{2}\right) \frac{2 c_{2}}{M \sqrt{\Lambda}}+c_{1} \frac{\left(1-\mu^{2}\right)^{\frac{s+2}{2}}}{\mu \Lambda^{\frac{s+2}{2}}}} \\
& +\frac{1}{s \Lambda} \int_{1 / \sqrt{1+\Lambda}}^{1} \mathrm{~d} \mu \frac{\left(1-\mu^{2}\right)^{2}}{\mu^{s-1}\left(1-\mu^{2}\right) \frac{2 c_{2}}{M \sqrt{\Lambda}}+c_{1} \frac{\left(1-\mu^{2}\right)^{\frac{s+2}{2}}}{\mu \Lambda^{\frac{s+2}{2}}}}
\end{aligned}
$$

which can be written as

$$
\begin{aligned}
g^{\mathrm{F}}\left(\Lambda \gg \epsilon^{-2} \gg 1, s\right) & \approx 2 \epsilon^{2-s}+\frac{1}{2 s c_{1}} \Lambda^{\frac{s-2}{2}}+\frac{1}{2-s}-\frac{1}{4-s} \\
& \approx \frac{2}{(2-s)(4-s)} .
\end{aligned}
$$

Table 6 summarizes the derived asymptotic behaviour.

\section{Transport parameters for mixed turbulence}

After having derived the individual momentum diffusion coefficients $A^{\mathrm{A}}, A^{\mathrm{F}}$ and the Fokker-Planck coefficients, it is straightforward to determine the transport parameters for the mixed turbulence once the relevant plasma parameters are specified. As an illustrative example we assume equal intensity and identical plasma and anisotropy parameters for shear Alfvén waves and fast magnetosonic waves, i.e.

$$
\begin{aligned}
& \delta B=\delta B^{\mathrm{A}}=\delta B^{\mathrm{F}}, \\
& s=s^{\mathrm{A}}=s^{\mathrm{F}}, \\
& k_{\min }=k_{\min }^{\mathrm{A}}=k_{\text {min }}^{\mathrm{F}} \\
& \Lambda=\Lambda^{\mathrm{A}}=\Lambda^{\mathrm{F}} .
\end{aligned}
$$

The application to other plasma turbulence mixtures is obvious.

In case of the illustrative example (66) the Fokker-Planck coefficient $D_{\mu \mu}^{\mathrm{MIX}}$ can be written as a sum of the Fokker-Planck coefficients from shear Alfvén waves and fast magnetosonic waves

$D_{\mu \mu}^{\mathrm{MIX}}=D_{\mu \mu}^{\mathrm{A}}+D_{\mu \mu}^{\mathrm{F}}$.

\subsection{The momentum diffusion coefficient $A^{\mathrm{MIX}}$}

With Eq. (67) we obtain

$A^{\mathrm{MIX}}=A^{\mathrm{A}}+A^{\mathrm{F}}$
Table 7. Anisotropy function $h^{\mathrm{MIX}}$ for mixed turbulence.

\begin{tabular}{l|l}
\hline \hline Anisotropy parameter $\Lambda$ & $h^{\mathrm{MIX}}(\Lambda, \epsilon, s)$ \\
\hline$\epsilon^{2} \ll \Lambda \ll 1$ & $c_{3}(s) \frac{\ln \epsilon^{-1}+\ln \Lambda^{1 / 2}}{\sqrt{\Lambda}}$ \\
$\Lambda=1$ & $c_{1}(s) \ln \epsilon^{-1}$ \\
$\Lambda \gg 1$ & $2\left(\frac{1}{s}-\frac{1}{s+2}\right)$ \\
\hline
\end{tabular}

which can be written as

$A^{\mathrm{MIX}}=\frac{\pi}{2}(s-1) \frac{(\delta B)^{2}}{B_{0}^{2}}\left(k_{\min } R_{L}\right)^{s-1} \frac{v \epsilon^{2} p^{2}}{R_{L}} h^{\mathrm{MIX}}(\Lambda, \epsilon, s)$

with

$$
\begin{aligned}
h^{\mathrm{MIX}}(\Lambda, \epsilon, s) & =h^{\mathrm{A}}(\Lambda, s)+h^{\mathrm{F}}(\Lambda, \epsilon, s) \\
& =h^{\mathrm{A}}(\Lambda, s)+h^{\mathrm{G}}(\Lambda, s)+h^{\mathrm{T}}(\Lambda, \epsilon, s) .
\end{aligned}
$$

With $h^{\mathrm{A}}$ from Table 1 and $h^{\mathrm{F}}$ from Table 5 we find for the function $h^{\mathrm{MIX}}$ the results listed in Table 7. As can be seen

$$
\begin{aligned}
A^{\mathrm{MIX}}(\Lambda \gg 1) & \approx A^{\mathrm{A}}(\Lambda \gg 1)+A^{\mathrm{G}}(\Lambda \gg 1) \\
& \approx 2 A^{\mathrm{G}}(\Lambda \gg 1) \\
A^{\mathrm{MIX}}(\Lambda=1) & \approx A^{\mathrm{T}}(\Lambda \approx 1)
\end{aligned}
$$$$
A^{\mathrm{MIX}}\left(\epsilon^{2} \ll \Lambda \ll 1\right) \approx A^{\mathrm{T}}\left(\epsilon^{2} \ll \Lambda \ll 1\right) .
$$

If $\epsilon^{2} \ll \Lambda \leq 1$ the main contribution to momentum diffusion in mixed turbulence (66) comes from transit-time damping of fast magnetosonic waves, whereas for $\Lambda \gg 1$ the two gyroresonance contributions are dominant.

\subsection{The spatial diffusion coefficient $\kappa^{\mathrm{MIX}}$}

The spatial diffusion coefficient in the mixed turbulence (66) can be written as

$$
\begin{aligned}
& \kappa^{\mathrm{MIX}}=\frac{v^{2}}{4} \int_{0}^{\epsilon} \mathrm{d} \mu \frac{1}{D_{\mu \mu}^{\mathrm{G}}(\mu \ll \epsilon)+D_{\mu \mu}^{\mathrm{A}}(\mu \ll \epsilon)} \\
&+\frac{v^{2}}{4} \int_{\epsilon}^{1} \mathrm{~d} \mu \frac{\left(1-\mu^{2}\right)}{D_{\mu \mu}^{\mathrm{G}}(\mu \gg \epsilon)+D_{\mu \mu}^{\mathrm{A}}(\mu \gg \epsilon)+D_{\mu \mu}^{\mathrm{T}}(\mu \gg \epsilon)} .
\end{aligned}
$$

Now we restrict our calculations to $\Lambda \ll \epsilon^{-2}$. To continue we must compare the Fokker-Planck coefficient for Alfvén waves $D_{\mu \mu}^{\mathrm{A}}$ with the gyroresonance contribution from the Fokker-Planck coefficient for fast magnetosonic waves $D_{\mu \mu}^{\mathrm{G}}$.

We start with the case $\mu \gg \epsilon$. Here we have (see Sect. 2):

$D_{\mu \mu}^{\mathrm{A}}(\mu \gg \epsilon) \propto \mu^{s+1} \cdot\left\{\begin{array}{ccc}\frac{M^{2}}{s \Lambda} & \text { for } & M^{2} \ll \Lambda \\ \text { const. } & \text { for } & \Lambda \ll M^{2} \ll 1 \\ \text { const. } & \text { for } & \Lambda \ll M^{2}, M^{2} \gg 1\end{array}\right.$

and according to Sect. 3:

$D_{\mu \mu}^{\mathrm{G}}(\mu \gg \epsilon)$

$$
\propto \mu^{s+1} \cdot\left\{\begin{array}{ccc}
\frac{M^{2}}{s \Lambda} & \text { for } & M^{2} \ll \Lambda \\
\frac{c_{2} M}{\sqrt{\Lambda}} \gg 1 & \text { for } & \Lambda \ll M^{2} \ll 1 \\
\frac{2 c_{2} M}{\sqrt{\Lambda}} \gg 1 & \text { for } & \Lambda \ll M^{2}, M^{2} \gg 1 .
\end{array}\right.
$$


Therefore it is easy to see that for all cases of $\Lambda$ and $M$ we have

$D_{\mu \mu}^{\mathrm{A}}(\mu \gg \epsilon) \leq D_{\mu \mu}^{\mathrm{G}}(\mu \gg \epsilon)$.

Comparing the Fokker-Planck coefficients for the case $\mu \ll \epsilon$, we can use (see Sect. 2)

$D_{\mu \mu}^{\mathrm{A}}(\mu \ll \epsilon) \propto \epsilon^{s+1}$,

and according to Sect. 3

$D_{\mu \mu}^{\mathrm{G}}(\mu \ll \epsilon) \propto \epsilon^{s} \cdot\left\{\begin{array}{ccc}\frac{c_{2}}{2 \Lambda^{(s+1) / 2}} & \text { for } & \Lambda \ll 1 \\ \frac{3 \zeta(s+1)}{4} & \text { for } & \Lambda=1 \\ \frac{c_{2}}{\sqrt{\Lambda}} & \text { for } & \epsilon^{-2} \gg \Lambda \gg 1 .\end{array}\right.$

Consequently, for all cases

$D_{\mu \mu}^{\mathrm{A}}(\mu \ll \epsilon) \ll D_{\mu \mu}^{\mathrm{G}}(\mu \ll \epsilon)$.

Hence, we obtain for the spatial diffusion coefficient in the mixed turbulence (66)

$\kappa^{\mathrm{MIX}} \approx \frac{v^{2}}{4} \int_{0}^{\epsilon} \frac{\mathrm{d} \mu}{D_{\mu \mu}^{\mathrm{G}}(\mu \ll \epsilon)} \approx \kappa^{\mathrm{F}}$.

The contribution from shear Alfvén waves and the transit-time damping contribution from fast magnetosonic waves are negligibly small in all cases. The spatial diffusion coefficient $\kappa^{\mathrm{MIX}}$ is dominated by the gyroresonance contribution from fast magnetosonic turbulence.

\section{Rigidity dependence of the transport parameters}

Here we calculate the rigidity dependence of the transport parameters. We introduce the rigidity $r$ and the parameter $r_{0}$

$r=\frac{p c}{|q|}$

$r_{0}=\frac{m c^{2}}{|q|}$,

so that

$R_{L} k_{\min }=\frac{k_{\min }}{B_{0}} r$

$\epsilon=\frac{v_{\mathrm{A}}}{v}=\frac{v_{\mathrm{A}}}{c} \frac{\sqrt{r_{0}^{2}+r^{2}}}{r}$.

The restriction $\epsilon \ll 1$ implies

$r \gg \frac{r_{0} v_{\mathrm{A}}}{\sqrt{c^{2}-v_{\mathrm{A}}^{2}}} \approx \frac{v_{\mathrm{A}}}{c} r_{0}$.

For electrons and positrons $r_{0}=0.511 \mathrm{MV}$, while for protons $r_{0}=938 M V$. If we adopt $\frac{v_{\mathrm{A}}}{c} \approx 10^{-4}$ the rigidity restriction reads

$r\left(\mathrm{e}^{\mp}\right) \gg 10^{-4} M V$,

$r\left(\mathrm{p}^{+}\right) \gg 10^{-1} M V$,

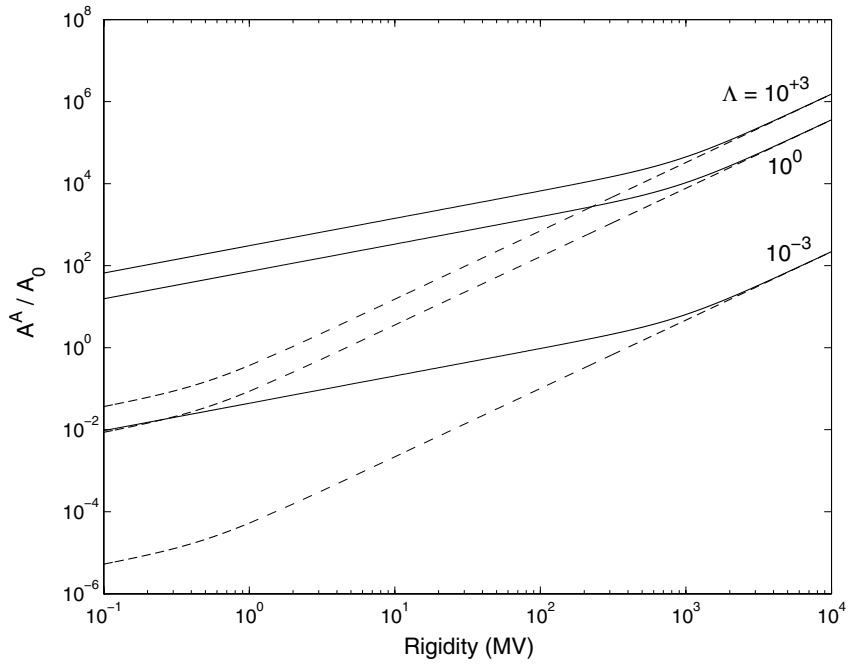

Fig. 1. The momentum diffusion coefficient for shear Alfvén waves $A^{\mathrm{A}}$ for protons (solid lines), electrons and positrons (dashed lines). We show the results for different values of the anisotropy parameter $\Lambda$.

which are assumed in the following analysis. Moreover, the assumption $R_{L} k_{\min } \ll 1$ becomes

$r \ll \frac{B_{0}}{k_{\min }} \approx 10^{4} M V$

for all particles, if we use $k_{\min } \approx 10^{-10} \frac{1}{m}$.

\subsection{The momentum diffusion coefficient $A^{i}$}

For Alfvén waves we use the results from TLS where we have shown that

$\frac{A^{\mathrm{A}}}{A_{0}}=\sqrt{\left(\frac{r_{0}}{M V}\right)^{2}+\left(\frac{r}{M V}\right)^{2}}\left(\frac{r}{M V}\right)^{s-1} \cdot h^{\mathrm{A}}(\Lambda, s)$

with

$A_{0}=\frac{\pi}{2}(s-1) \frac{k_{\min }^{s-1}}{B_{0}^{s} c}\left(q \frac{v_{\mathrm{A}} \delta B}{c}\right)^{2}(M V)^{s}$.

If we do the corresponding calculations for fast magnetosonic waves we find a similar result, but there the anisotropy function $h$ is also a function of $\epsilon$, and therefore through Eq. (81) also a function of particle rigidity $r$. It is possible to write for all turbulence models

$\frac{A^{i}}{A_{0}}=\sqrt{\left(\frac{r_{0}}{M V}\right)^{2}+\left(\frac{r}{M V}\right)^{2}}\left(\frac{r}{M V}\right)^{s-1} \cdot h^{i}(\Lambda, \epsilon, s)$

where $A_{0}$ is the same function for all models. For $h^{\mathrm{A}}$ we can use Table 1 , for $h^{\mathrm{F}}$ we can use Table 5 and for $h^{\mathrm{MIX}}$ we can use Table 7. In Fig. 1 we show the resulting momentum diffusion coefficients for shear Alfvén waves and in Fig. 2 for fast magnetosonic waves, respectively.

\subsection{The spatial diffusion coefficient $\kappa^{i}$}

Restricting our analysis to $\Lambda \ll \epsilon^{-2}$ we have

$\frac{\kappa^{i}}{\kappa_{0}}=\frac{\left(\frac{r}{M V}\right)^{3-s}}{\sqrt{\left(\frac{r_{0}}{M V}\right)^{2}+\left(\frac{r}{M V}\right)^{2}}} \cdot g^{i}(\Lambda, \epsilon, s)$ 


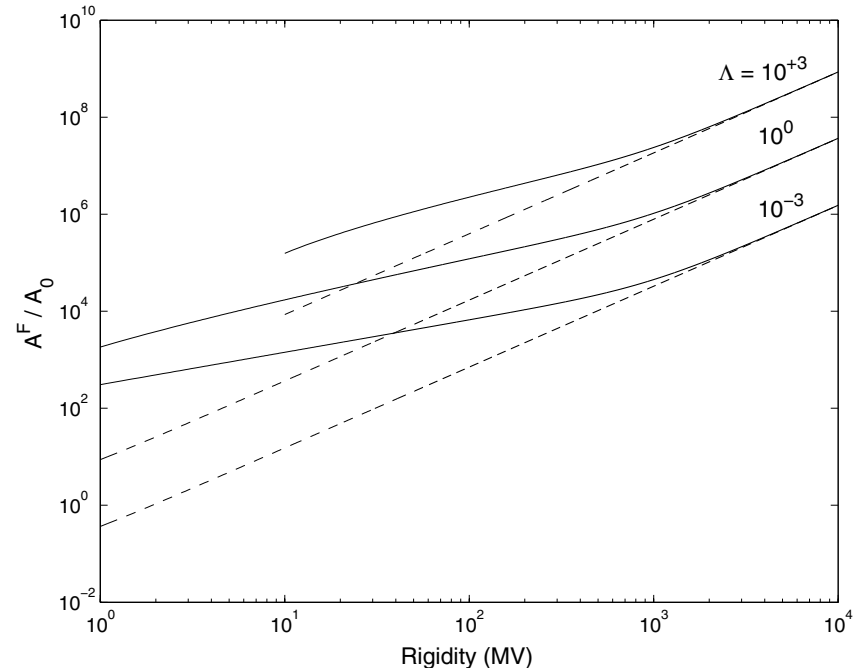

Fig. 2. The momentum diffusion coefficient for fast magnetosonic waves $A^{\mathrm{F}}$ for protons (solid lines), electrons and positrons (dashed lines). We show the results for different values of the anisotropy parameter $\Lambda$.

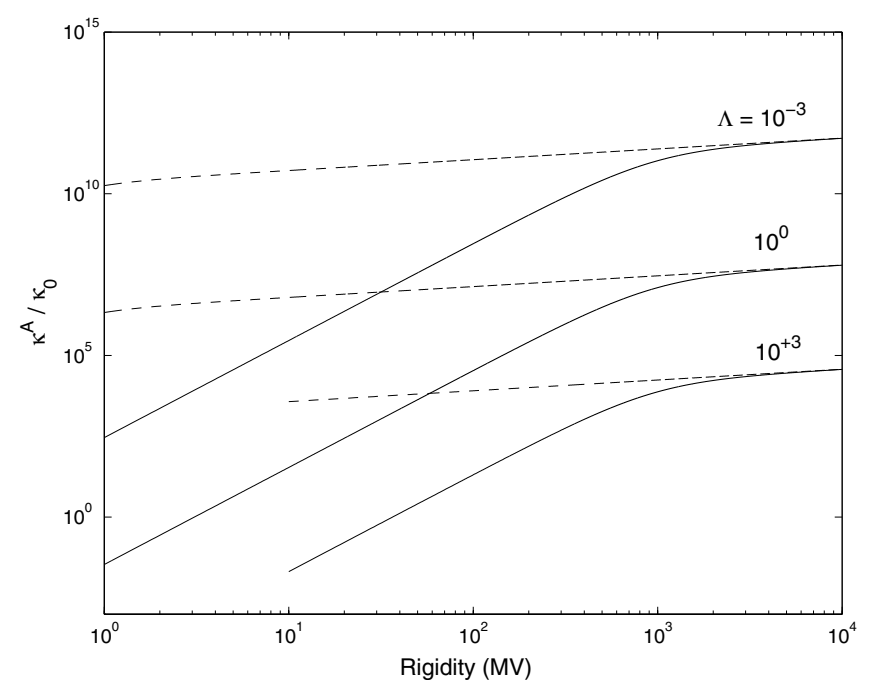

Fig. 3. The parallel spatial diffusion coefficient for shear Alfvén waves $\kappa^{\mathrm{A}}$ for protons (solid lines), electrons and positrons (dashed lines). We show the results for different values of the anisotropy parameter $\Lambda$.

with

$\kappa_{0}=\frac{1}{2 \pi(s-1)} \frac{c B_{0}^{s}}{\delta B^{2} k_{\min }^{s-1}}(M V)^{2-s}$.

For $g^{\mathrm{A}}$ we can use Table 2, for $g^{\mathrm{F}}$ we can use Table 6 and we know that $g^{\text {MIX }} \approx g^{\mathrm{F}}$. In Fig. 3 we show the spatial diffusion coefficient for shear Alfvén waves and in Fig. 4 for fast magnetosonic waves, which also gives the spatial diffusion coefficient in case for the mixed turbulence (66).

\subsection{The parallel mean free path $\lambda^{i}$}

The parallel mean free path is given by

$\lambda^{i}=\frac{3}{v} \kappa^{i}$.

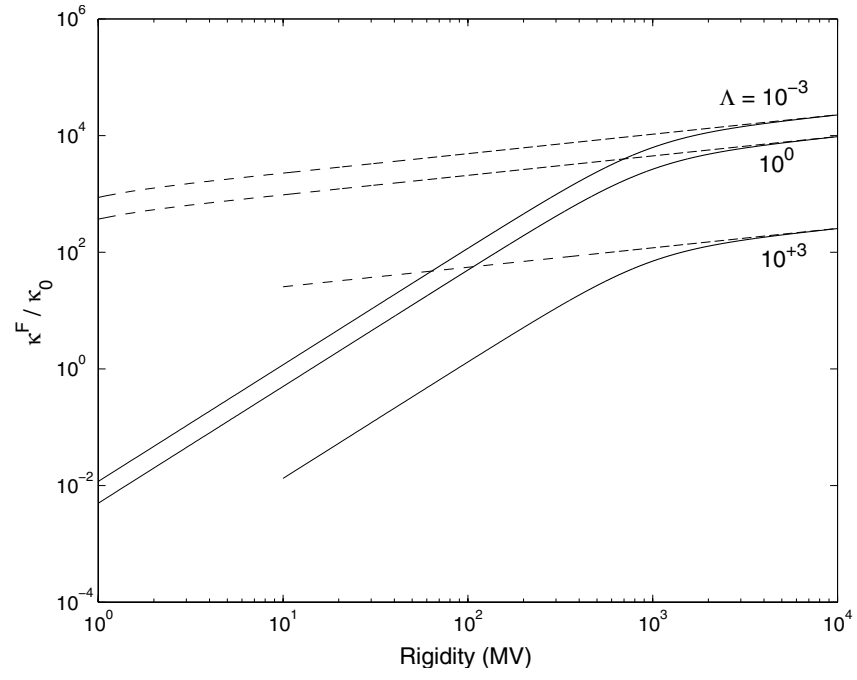

Fig. 4. The parallel spatial diffusion coefficient for fast magnetosonic waves $\kappa^{\mathrm{F}}$ for protons (solid lines), electrons and positrons (dashed lines). We show the results for different values of the anisotropy parameter $\Lambda$.

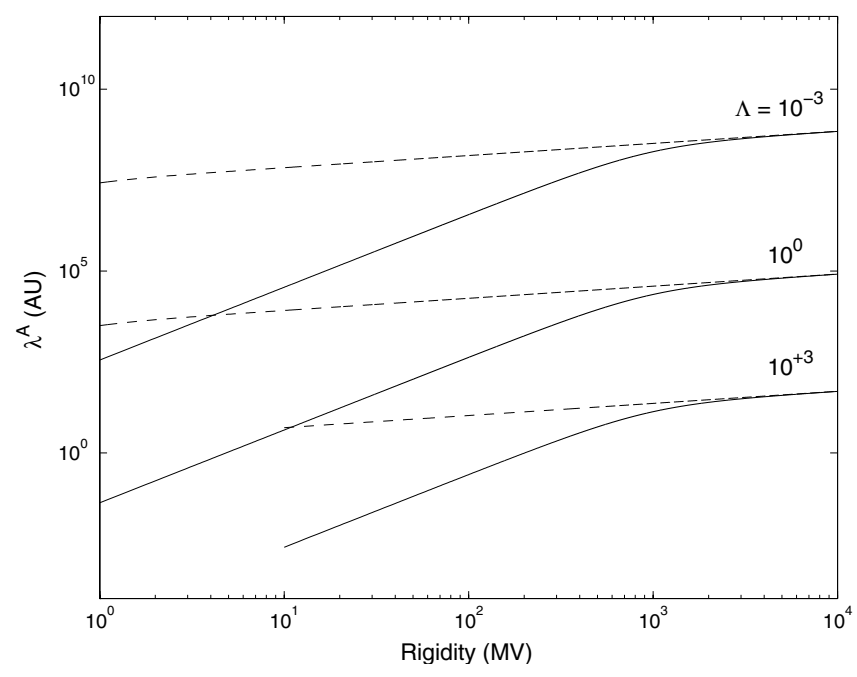

Fig. 5. The parallel mean free path for shear Alfvén waves $\lambda^{\mathrm{A}}$ for protons (solid lines), electrons and positrons (dashed lines). We show the results for different values of the anisotropy parameter $\Lambda$.

This can be written as

$\frac{\lambda^{i}}{\lambda_{0}}=\frac{\sqrt{\left(\frac{r_{0}}{M V}\right)^{2}+\left(\frac{r}{M V}\right)^{2}}}{\left(\frac{r}{M V}\right)} \cdot \frac{\kappa^{i}}{\kappa_{0}}=\left(\frac{r}{M V}\right)^{2-s} \cdot g^{i}(\Lambda, \epsilon, s)$

with

$\lambda_{0}=\frac{3 \kappa_{0}}{c}$

and we know that $\lambda^{\mathrm{MIX}} \approx \lambda^{\mathrm{F}}$. Figure 5 shows the parallel mean free path for shear Alfvén waves, while in Fig. 6 the mean free path for fast magnetosonic waves and therefore for the mixed turbulence (66) is shown. 


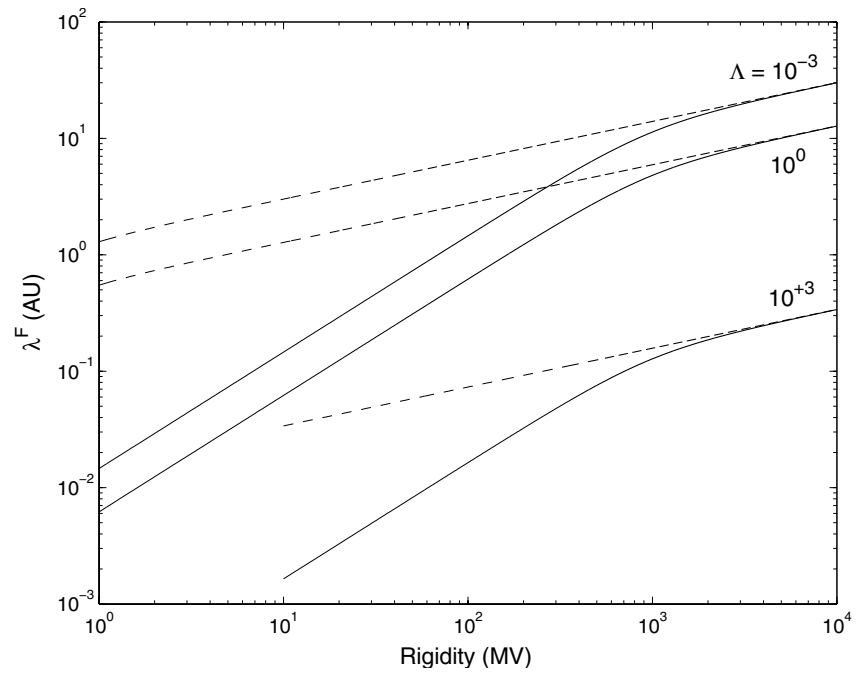

Fig. 6. The parallel mean free path for fast magnetosonic waves $\lambda^{\mathrm{F}}$ for protons (solid lines), electrons and positrons (dashed lines). We show the results for different values of the anisotropy parameter $\Lambda$.

\section{Summary and conclusions}

We have continued to evaluate the relevant cosmic ray transport parameters in the presence of anisotropic plasma wave turbulence. Using the estimates of the anisotropy parameter in the strongly parallel and perpendicular regimes, based on linear Landau damping balancing radiative loss in the diffuse interstellar medium, we have calculated the Fokker-Planck coefficients, the momentum $(A)$ and parallel spatial diffusion coefficient $(\kappa)$ and the parallel mean free path $(\lambda)$ of cosmic ray particles for a mixed plasma turbulence of shear Alfvén waves and fast magnetosonic waves. We elaborated in detail on the case of equal intensity, plasma and anisotropy parameters of shear Alfvénic and magnetosonic turbulence, but our formalism easily allows generalization to any intensity ratio of these plasma waves.

In the equal intensity case, regarding the influence of the anisotropy parameter $\Lambda$, we find that for strongly perpendicular turbulence $(\Lambda \ll 1) A \sim \Lambda^{-1 / 2}\left[\ln \epsilon^{-1}+\ln \Lambda^{1 / 2}\right]$ and $\kappa \sim$ const. whereas for strongly parallel turbulence $(\Lambda \gg 1)$ we obtain $A \sim$ const. and $\kappa \sim \Lambda^{-1 / 2}$. Moreover, for the momentum diffusion coefficient we show that for a non-slab-like geometry the transit-time damping of the fast magnetosonic waves is dominant. In slab-like turbulence $(\Lambda \gg 1)$ both gyroresonance contributions are equal and dominate over transit-time damping. For the spatial diffusion coefficient and the mean free path we find that the Alfvén contribution can be neglected. The gyroresonance contribution of the fast magnetosonic waves controls the spatial diffusion coefficient and the parallel mean free path.

We established that the rigidity dependences of the momentum and parallel spatial diffusion coefficients for general values of $\Lambda \neq 1$ are the same as in the case of isotropic $(\Lambda=1)$ turbulence discussed earlier by Schlickeiser \& Miller (1998); however, the absolute values of these transport parameters are strongly dependent on the value of $\Lambda$. As indicated above the value of momentum diffusion coefficient increases $\sim \Lambda^{-1 / 2}$ for strongly perpendicular $(\Lambda \ll 1)$ turbulence, whereas the value of the parallel diffusion coefficient decreases $\sim \Lambda^{-1 / 2}$ for strongly parallel $(\Lambda \gg 1)$ turbulence.

We also demonstrated that in general the parameter $\epsilon$ has a strong influence on the results of transport parameters. Therefore the plasma wave dispersion relation and the turbulent electrical fields cannot be neglected if one calculates spatial and momentum diffusion coefficients in anisotropic turbulence geometry.

Our results have direct implications for the cosmic ray transport parameters inferred from the observations of galactic cosmic rays: in particular the cosmic ray anisotropy and the diffusive cosmic ray escape time, inferred from the decay of radioactive cosmic ray isotopes and the secondary-to-primaryratio, are related to the spatial diffusion coefficients, while the rate of stochastic acceleration is determined by the momentum diffusion coefficient. However, a detailed discussion of these implications is premature, before the role of perpendicular spatial diffusion in the mixed plasma wave turbulence fields has been clarified. Moreover, it also is useful to extend our study to arbitrary intensity ratios of Alfvénic and magnetosonic waves and to investigate the effect of steeper $(s>2)$ turbulence power spectra.

A further future study concerns the cosmic ray rigidity range. For all the calculations done sofar in the three papers of this series we used the restriction $R_{L} k_{\min } \ll 1$. But it is also very important to consider the opposite limit $\left(R_{L} k_{\min } \gg 1\right)$ for ultrahigh cosmic ray energies. These topics will be the subject of future work.

Acknowledgements. This work was partially supported by the Deutsche Forschungsgemeinschaft through Sonderforschungsbereich 591. A.S. acknowledge support by the National Science Foundation under grant ATM-0000315.

\section{References}

Abramowitz, M., \& Stegun, I. A. 1972, Handbook of Mathematical Functions, National Bureau of Standards, Washington

Bieber, J. W., Matthaeus, W. H., Smith, C. W., et al. 1994, ApJ, 420, 294

Chandran, B. 2001, Phys. Rev. Lett., 85, 4656

Goldreich, P., \& Sridhar, S. 1995, ApJ, 438, 763

Lerche, I., \& Schlickeiser, R. 2001a, A\&A, 366, 1008

Lerche, I., \& Schlickeiser, R. 2001b, A\&A, 378, 279 (LS)

Magnus, W., Oberhettinger, F., \& Soni, R. P. 1966, Formulas and Theorems for the Special Functions of Mathematical Physics (Berlin, Heidelberg, New York: Springer-Verlag)

Rickett, B. J. 1990, ARA\&A, 28, 561

Schlickeiser, R., \& Miller, J. A. 1998, ApJ, 492, 352

Schlickeiser, R. 2002, Cosmic Ray Astrophysics (Berlin Heidelberg: Springer-Verlag)

Shalchi, A., \& Schlickeiser, R. 2004, ApJ, 604, 861

Spangler, S. R. 1991, ApJ, 376, 540

Teufel, A., Lerche, I., \& Schlickeiser, R., 2003, A\&A, 397, 777 (TLS)

Yan, H., \& Lazarian, A. 2002, Phys. Rev. Lett., 89, 1102

Watson, G. N. 1966, A treatise on the Theory of Bessel Functions (Cambridge University Press) 
A. Shalchi and R. Schlickeiser: Cosmic rays in anisotropic turbulence, Online Material p 1

\section{Online Material}




\section{Appendix A: Calculation of $D_{\mu \mu}^{\mathrm{G}}(\mu \gg \epsilon)$ for fast magnetosonic waves}

From LS we know that

$D_{\mu \mu}^{\mathrm{G}}(\mu \gg \epsilon)=\frac{2 \pi^{2} v R_{L}^{s-2}\left(1-\mu^{2}\right)|\mu|^{s-1} I_{0}}{B_{0}^{2}} W(\Lambda, s, \mu)$

with

$$
\begin{aligned}
W_{l}^{\mathrm{G}}(\Lambda, s, \mu)= & \sum_{n=1}^{\infty} \int_{0}^{\infty} \mathrm{d} x \frac{x+2}{x+1} \frac{1}{[1+\Lambda x]^{(s+2) / 2}} \\
& \times\left[J_{n}^{\prime}(n M \sqrt{x})\right]^{2}
\end{aligned}
$$

and with

$$
I_{0}=\frac{(\delta B)^{2}(s-1) k_{\min }^{s-1}}{4 \pi J(\Lambda)}
$$

under the condition $R_{L} k_{\text {min }} \ll 1$. We split the integral as

$$
\begin{aligned}
W_{l}^{\mathrm{G}}(\Lambda, s, \mu)= & \int_{0}^{M^{-2}} \mathrm{~d} x \frac{x+2}{x+1} \frac{1}{[1+\Lambda x]^{(s+2) / 2}} \\
& \times \sum_{n=1}^{\infty}\left[J_{n}^{\prime}(n M \sqrt{x})\right]^{2} \\
& +\int_{M^{-2}}^{\infty} \mathrm{d} x \frac{x+2}{x+1} \frac{1}{[1+\Lambda x]^{(s+2) / 2}} \\
& \times \sum_{n=1}^{\infty}\left[J_{n}^{\prime}(n M \sqrt{x})\right]^{2}
\end{aligned}
$$

and approximate the two sums.

To approximate the first sum we use the Kapteyn series from Appendix C

$\sum_{n=1}^{\infty} n^{-2} J_{n}^{2}(n z)=\frac{1}{4}-\frac{z^{2}}{8}$

so that

$\sum_{n=1}^{\infty} n^{-s} J_{n}^{\prime 2}(n M \sqrt{x}) \approx \sum_{n=1}^{\infty} n^{-2} J_{n}^{\prime 2}(n M \sqrt{x})=\frac{1}{4}-\frac{M^{2} x}{8}$.

The second sum can be approximated with

$\sum_{n=1}^{\infty} n^{-s} J_{n}^{\prime 2}(n M \sqrt{x}) \approx \sum_{n=1}^{\infty} n^{-s} \frac{1}{\pi n M \sqrt{x}}=\frac{\zeta(s+1)}{\pi M \sqrt{x}}$

where we neglect the oscillating part in the approximation of the Bessel function for large arguments (Abramowitz \& Stegun 1972). We then obtain

$$
\begin{aligned}
W_{l}^{\mathrm{G}}(\Lambda, s, \mu)= & \frac{1}{4} \int_{0}^{M^{-2}} \mathrm{~d} x \frac{x+2}{x+1} \frac{1}{[1+\Lambda x]^{(s+2) / 2}} \\
& -\frac{M^{2}}{8} \int_{0}^{M^{-2}} \mathrm{~d} x \frac{x+2}{x+1} \frac{x}{[1+\Lambda x]^{(s+2) / 2}} \\
& +\frac{\zeta(s+1)}{\pi M} \int_{M^{-2}}^{\infty} \mathrm{d} x \frac{x+2}{x+1} \frac{x^{-1 / 2}}{[1+\Lambda x]^{(s+2) / 2}} .
\end{aligned}
$$

We write this as

$W_{l}^{\mathrm{G}}(\Lambda, s, \mu)=\frac{1}{4} K_{1}-\frac{M^{2}}{8} K_{2}+\frac{\zeta(s+1)}{\pi M} K_{3}$ with the integrals

$$
\begin{aligned}
& K_{1}=\int_{0}^{M^{-2}} \mathrm{~d} x \frac{x+2}{x+1} \frac{1}{[1+\Lambda x]^{(s+2) / 2}}, \\
& K_{2}=\int_{0}^{M^{-2}} \mathrm{~d} x \frac{x+2}{x+1} \frac{x}{[1+\Lambda x]^{(s+2) / 2}}
\end{aligned}
$$

and

$$
K_{3}=\int_{M^{-2}}^{\infty} \mathrm{d} x \frac{x+2}{x+1} \frac{x^{-1 / 2}}{[1+\Lambda x]^{(s+2) / 2}} .
$$

We consider the three integrals in the limits of small and large $M^{2}$ and $\Lambda M^{-2}$ in turn.

\section{A.1. The case of $M^{2} \ll 1$ and $M^{2} \ll \wedge$}

Here $M^{-2} \gg 1$ so that

$$
\begin{aligned}
K_{1} & \approx 2 \int_{0}^{M^{-2}} \mathrm{~d} x[1+\Lambda x]^{-\frac{s+2}{2}} \\
& =\frac{4}{s \Lambda}\left[1-\left(1+\Lambda M^{-2}\right)^{-s / 2}\right] \\
K_{2} & \approx 2 \int_{0}^{M^{-2}} \mathrm{~d} x x[1+\Lambda x]^{-\frac{s+2}{2}} \\
& =\frac{1}{M^{4}}{ }_{2} F_{1}\left(\frac{s+2}{2}, 2,3,-\Lambda M^{-2}\right) \\
K_{3} & \approx \int_{M^{-2}}^{\infty} \frac{\mathrm{d} x}{\sqrt{x}}[1+\Lambda x]^{-\frac{s+2}{2}} \\
& =\frac{2 M^{s+1}}{(s+1) \Lambda^{(s+2) / 2}}{ }_{2} F_{1}\left(\frac{s+2}{2}, \frac{s+1}{2}, \frac{s+3}{2}, \frac{-M^{2}}{\Lambda}\right) .
\end{aligned}
$$

For $W_{l}^{\mathrm{G}}$ we then obtain approximately

$W_{l}^{\mathrm{G}}\left(M^{2} \ll 1, \Lambda, s\right) \approx \frac{1}{s \Lambda}-\frac{1}{s \Lambda}\left[1+\Lambda M^{-2}\right]^{-s / 2}$

$-\frac{1}{8 M^{2}}{ }_{2} F_{1}\left(\frac{s+2}{2}, 2 ; 3 ;-\Lambda M^{-2}\right)$

$+\frac{2 \zeta(s+1)}{(s+1) \pi} \frac{M^{s}}{\Lambda^{(s+2) / 2}}{ }_{2} F_{1}\left(\frac{s+2}{2}, \frac{s+1}{2} ; \frac{s+3}{2} ;-\frac{M^{2}}{\Lambda}\right)$.

In the limit $\Lambda M^{-2} \gg 1$ we find

$W_{l}^{\mathrm{G}}\left(M^{2} \ll 1, M^{2} \ll \Lambda, s\right) \approx \frac{1}{s \Lambda}$.

To derive the last equations we have used the following approximations and relations for the hypergeometric functions (Magnus et al. 1966):

$$
\begin{aligned}
& { }_{2} F_{1}(a, b ; c ; z \ll 1) \approx{ }_{2} F_{1}(a, b ; c ; 0)=1, \\
& { }_{2} F_{1}(a, b ; c ; z)=(1-z)^{-a}{ }_{2} F_{1}\left(a, c-b ; c ; \frac{z}{z-1}\right), \\
& { }_{2} F_{1}(a, b ; c ; z)=(1-z)^{-b}{ }_{2} F_{1}\left(b, c-a ; c ; \frac{z}{z-1}\right),
\end{aligned}
$$

and if $c>a+b$

$$
{ }_{2} F_{1}(a, b ; c ; 1)=\frac{\Gamma(c) \Gamma(c-a-b)}{\Gamma(c-a) \Gamma(c-b)} .
$$


A. Shalchi and R. Schlickeiser: Cosmic rays in anisotropic turbulence, Online Material p 3

\section{A.2. The case of $\wedge \ll M^{2} \ll 1$}

Here we obtain for $W_{l}^{\mathrm{G}}$ approximately

$W_{l}^{\mathrm{G}}\left(\Lambda \ll M^{2} \ll 1\right) \approx \frac{c_{2}}{\sqrt{\Lambda} M}$

with

$c_{2}=\frac{2 \zeta(s+1) \Gamma\left(\frac{s+3}{2}\right)}{\sqrt{\pi}(s+1) \Gamma\left(\frac{s+2}{2}\right)}$

where we have used the same relations and approximations (Eqs. (A.18)-(A.21)) as in the last case.

\section{A.3. The case of $1 \ll M^{2} \ll \wedge$}

In this case $M^{-2} \ll 1$ is a small quantity and the three integrals are approximately

$$
\begin{aligned}
K_{1} & \approx 2 \int_{0}^{M^{-2}} \mathrm{~d} x[1+\Lambda x]^{-\frac{s+2}{2}} \\
& =\frac{4}{s \Lambda}\left[1-\left(1+\Lambda M^{-2}\right)^{-s / 2}\right] \\
K_{2} & \approx 2 \int_{0}^{M^{-2}} \mathrm{~d} x x[1+\Lambda x]^{-\frac{s+2}{2}} \\
& =\frac{1}{M^{4}}{ }_{2} F_{1}\left(\frac{s+2}{2}, 2,3,-\Lambda M^{-2}\right) \\
K_{3} & \approx 2 \int_{M^{-2}}^{\infty} \frac{\mathrm{d} x}{\sqrt{x}}[1+\Lambda x]^{-\frac{s+2}{2}} \\
& \approx \frac{2 M^{s+1}}{(s+1) \Lambda^{(s+2) / 2}}{ }_{2} F_{1}\left(\frac{s+2}{2}, \frac{s+1}{2}, \frac{s+3}{2}, \frac{-M^{2}}{\Lambda}\right)
\end{aligned}
$$

and we then obtain

$$
\begin{aligned}
& W_{l}^{\mathrm{G}}\left(M^{2} \gg 1, \Lambda, s\right) \approx \frac{1}{s \Lambda}-\frac{1}{s \Lambda}\left[1+\Lambda M^{-2}\right]^{-s / 2} \\
& -\frac{1}{8 M^{2}}{ }_{2} F_{1}\left(\frac{s+2}{2}, 2 ; 3 ;-\Lambda M^{-2}\right) \\
& +\frac{4 \zeta(s+1)}{(s+1) \pi} \frac{M^{s}}{\Lambda^{(s+2) / 2}}{ }_{2} F_{1}\left(\frac{s+2}{2}, \frac{s+1}{2} ; \frac{s+3}{2} ;-\frac{M^{2}}{\Lambda}\right)
\end{aligned}
$$

in the limit $\Lambda M^{-2} \gg 1$ and $\Lambda \gg 1$. With Eqs. (A.18)-(A.21) we obtain

$W_{l}^{\mathrm{G}}\left(1 \ll M^{2} \ll \Lambda\right) \approx \frac{1}{s \Lambda}$.

\section{A.4. The case of $M^{2} \gg 1$ and $M^{2} \gg \Lambda$}

In the last case we find for $W_{l}$ approximately

$W_{l}^{\mathrm{G}}\left(M^{2} \gg 1, M^{2} \gg \Lambda\right) \approx \frac{2 c_{2}}{\sqrt{\Lambda} M}$

where we have used the same relations and approximations as in the first three cases.

\section{Appendix B: Calculation of $D_{\mu \mu}^{\mathrm{G}}(\mu \ll \epsilon)$ for fast magnetosonic waves}

From LS we know that

$$
\begin{aligned}
D_{\mu \mu}^{\mathrm{G}}(\mu \ll \epsilon) & =\frac{4 \pi^{2} v^{2}}{R_{L}^{2} B_{0}^{2} v_{A}} I_{0} \sum_{n=1}^{\infty} \int_{0}^{1} \mathrm{~d} \eta \frac{1+\eta^{2}}{\left[\eta^{2}+\Lambda\left(1-\eta^{2}\right)\right]^{(s+2) / 2}} \\
& \times \int_{k_{\min }}^{\infty} \mathrm{d} k k^{-s} \delta\left(k-\frac{n \Omega}{v_{A}}\right) J_{n}^{2}\left(k R_{L} \sqrt{1-\eta^{2}}\right) .
\end{aligned}
$$

Now we restrict our analysis to $R_{L} k_{\min } \ll 1$. With Eq. (A.3) we then find

$D_{\mu \mu}^{\mathrm{G}}(\mu \ll \epsilon)=\frac{\pi(s-1) R_{L}^{s-2} v \epsilon^{s-1} k_{\min }^{s-1}}{J(\Lambda)} \frac{(\delta B)^{2}}{B_{0}^{2}} W_{s}(\Lambda, \epsilon, s)$

with

$$
\begin{aligned}
W_{s}^{\mathrm{G}}(\Lambda, \epsilon, s)= & \int_{0}^{\sqrt{1-\epsilon^{2}}} \mathrm{~d} \eta \frac{1+\eta^{2}}{\left[\eta^{2}+\Lambda\left(1-\eta^{2}\right)\right]^{(s+2) / 2}} \\
& \times \sum_{n=1}^{\infty} n^{-s} J_{n}^{2}\left(\frac{n}{\epsilon} \sqrt{1-\eta^{2}}\right) \\
& +\int_{\sqrt{1-\epsilon^{2}}}^{1} \mathrm{~d} \eta \frac{1+\eta^{2}}{\left[\eta^{2}+\Lambda\left(1-\eta^{2}\right)\right]^{(s+2) / 2}} \\
& \times \sum_{n=1}^{\infty} n^{-s} J_{n}^{\prime 2}\left(\frac{n}{\epsilon} \sqrt{1-\eta^{2}}\right) .
\end{aligned}
$$

With the same approximations for the sums of Bessel functions as used in Appendix A we obtain

$$
\begin{aligned}
W_{s}^{\mathrm{G}}(\Lambda, \epsilon, s) \approx & \frac{\zeta(s+1) \epsilon}{\pi} \int_{0}^{\sqrt{1-\epsilon^{2}}} \mathrm{~d} \eta \frac{1+\eta^{2}}{\sqrt{1-\eta^{2}}} \\
& \times \frac{1}{\left[\eta^{2}+\Lambda\left(1-\eta^{2}\right)\right]^{(s+2) / 2}} \\
& +\frac{1}{8} \int_{\sqrt{1-\epsilon^{2}}}^{1} d \eta \frac{1+\eta^{2}}{\left[\eta^{2}+\Lambda\left(1-\eta^{2}\right)\right]^{(s+2) / 2}} \\
& \times\left(2-\frac{1-\eta^{2}}{\epsilon^{2}}\right) .
\end{aligned}
$$

We can express these integrals in terms of hypergeometric functions:

$$
\begin{aligned}
W_{s}^{\mathrm{G}}(\Lambda, \epsilon, s) \approx & \frac{\zeta(s+1) \epsilon}{2}{ }_{2} F_{1}\left(\frac{s+2}{2}, \frac{1}{2}, 1 ;-(\Lambda-1)\right) \\
& +\frac{\zeta(s+1) \epsilon}{4}{ }_{2} F_{1}\left(\frac{s+2}{2}, \frac{1}{2}, 2 ;-(\Lambda-1)\right) \\
& -\frac{2 \zeta(s+1) \epsilon^{2}}{\pi}{ }_{2} F_{1}\left(\frac{s+2}{2}, \frac{1}{2}, 2 ;-(\Lambda-1) \epsilon^{2}\right) \\
& +\frac{\epsilon^{2}}{4}{ }_{2} F_{1}\left(\frac{s+2}{2}, 1,2 ;-(\Lambda-1) \epsilon^{2}\right) \\
& -\frac{\epsilon^{2}}{16}{ }_{2} F_{1}\left(\frac{s+2}{2}, 2,3 ;-(\Lambda-1) \epsilon^{2}\right) .
\end{aligned}
$$

Now we consider special cases for $\Lambda$ and $\Lambda \epsilon^{2}$ : 
A. Shalchi and R. Schlickeiser: Cosmic rays in anisotropic turbulence, Online Material p 4

\section{B.1. Isotropic turbulence $\Lambda=1$}

Here we find with Eqs. (A.18)-(A.21) that

$W_{s}^{\mathrm{G}}(\Lambda=1, \epsilon, s) \approx \frac{3}{4} \zeta(s+1) \epsilon$

to lowest order in $\epsilon$.

\section{B.2. Strongly perpendicular turbulence $\Lambda \ll 1$}

Here we obtain approximately

$$
\begin{aligned}
W_{s}^{\mathrm{G}}(\Lambda & \ll 1, \epsilon, s) \approx \frac{\zeta(s+1) \epsilon}{2} \Lambda^{-(s+1) / 2}{ }_{2} F_{1}\left(-\frac{s}{2}, \frac{1}{2}, 1 ; 1\right) \\
& +\frac{\zeta(s+1) \epsilon}{4} \Lambda^{(1-s) / 2}{ }_{2} F_{1}\left(1-\frac{s}{2}, \frac{3}{2}, 2 ; 1\right) .
\end{aligned}
$$

If we use $\epsilon \ll 1$ and $\Lambda \ll 1$ we find for this case

$$
W_{s}^{\mathrm{G}}(\Lambda \ll 1, \epsilon, s) \approx \frac{\zeta(s+1) \epsilon}{2 \sqrt{\pi} \Lambda^{(s+1) / 2}} \frac{\Gamma\left(\frac{s+1}{2}\right)}{\Gamma\left(\frac{s+2}{2}\right)} .
$$

\section{B.3. Strongly parallel turbulence $1 \ll \Lambda \ll \epsilon^{-2}$}

In this case we can use approximations for the hypergeometric functions again to find that

$$
\begin{aligned}
W_{s}^{\mathrm{G}}\left(\Lambda \gg 1, \Lambda \epsilon^{2} \ll 1, \epsilon, s\right) \approx \frac{\zeta(s+1) \epsilon}{2 \sqrt{\Lambda}}{ }_{2} F_{1}\left(\frac{1}{2},-\frac{s}{2}, 1 ; 1\right) \\
+\frac{\zeta(s+1) \epsilon}{4 \sqrt{\Lambda}}{ }_{2} F_{1}\left(\frac{1}{2}, 1-\frac{s}{2}, 2 ; 1\right) .
\end{aligned}
$$

We can express the hypergeometric functions through $\Gamma$-functions and in the limit $\Lambda \epsilon^{2} \ll 1$ we obtain

$$
W_{s}^{\mathrm{G}}\left(\Lambda \gg 1, \Lambda \epsilon^{2} \ll 1, \epsilon, s\right) \approx \frac{\zeta(s+1) \epsilon}{\sqrt{\pi} \sqrt{\Lambda}} \frac{\Gamma\left(\frac{s+1}{2}\right)}{\Gamma\left(\frac{s+2}{2}\right)} .
$$

\section{B.4. Extremelly parallel turbulence $1 \ll \epsilon^{-2} \ll \Lambda$}

Here we find

$$
\begin{aligned}
W_{s}^{\mathrm{G}} & \left(\Lambda \gg 1, \Lambda \epsilon^{2} \ll 1, \epsilon, s\right) \approx \frac{\zeta(s+1) \epsilon}{\sqrt{\Lambda} \sqrt{\pi}} \frac{\Gamma\left(\frac{s+1}{2}\right)}{\Gamma\left(\frac{s+2}{2}\right)} \\
& -\frac{2 \zeta(s+1) \epsilon^{2}}{\pi}\left(1+\Lambda \epsilon^{2}\right)^{-1 / 2}{ }_{2} F_{1}\left(\frac{1}{2}, \frac{1-s}{2}, \frac{3}{2} ; 1\right) \\
+ & \frac{\epsilon^{2}}{4}\left(1+\Lambda \epsilon^{2}\right)^{-1} 2 F_{1}\left(1, \frac{2-s}{2}, 2 ; 1\right) \\
- & \frac{\epsilon^{2}}{16}\left(1+\Lambda \epsilon^{2}\right)^{-(s+2) / 2} 2 F_{1}\left(\frac{s+2}{2}, 1,3 ; 1\right)
\end{aligned}
$$

and we obtain for this case

$$
W_{s}^{\mathrm{G}}\left(\Lambda \gg 1, \Lambda \epsilon^{2} \ll 1, \epsilon, s\right) \approx \frac{1}{2 s \Lambda} .
$$

Appendix C: The Kapteyn series $\sum_{n=1}^{\infty} n^{-2} J_{n}^{\prime 2}(n z)$

With the Bessel function identities

$$
\left[2 J_{n}^{\prime}(n z)\right]^{2}=\left[J_{n-1}(n z)-J_{n+1}(n z)\right]^{2}
$$

and

$\left[\frac{2 n J_{n}(n z)}{n z}\right]^{2}=\left[J_{n-1}(n z)+J_{n+1}(n z)\right]^{2}$

we find

$J_{n}^{\prime 2}(n z)=\left(\frac{J_{n}(n z)}{z}\right)^{2}-J_{n-1}(n z) J_{n+1}(n z)$.

Therefore we obtain for the series

$$
\begin{aligned}
\sum_{n=1}^{\infty} n^{-2} J_{n}^{\prime 2}(n z)= & \frac{1}{z^{2}} \sum_{n=1}^{\infty} n^{-2} J_{n}^{2}(n z) \\
& -\sum_{n=1}^{\infty} n^{-2} J_{n-1}(n z) J_{n+1}(n z) .
\end{aligned}
$$

With the Kapteyn series (Watson 1966)

$\sum_{n=1}^{\infty} n^{-2} J_{n}^{2}(n z)=\frac{z^{2}}{4}$

and with (Watson 1966)

$J_{n-1}(n z) J_{n+1}(n z)=\frac{2}{\pi} \int_{0}^{\pi / 2} \mathrm{~d} \Phi J_{2 n}(2 n z \cos \Phi) \cos (2 \Phi)$

we obtain

$\sum_{n=1}^{\infty} n^{-2} J_{n}^{2}(n z)$

$$
=\frac{1}{4}-\frac{2}{\pi} \int_{0}^{\pi / 2} \mathrm{~d} \Phi \cos (2 \Phi) \sum_{n=1}^{\infty} n^{-2} J_{2 n}(2 n z \cos \Phi) .
$$

With (Watson 1966)

$\sum_{n=1}^{\infty} n^{-2} J_{2 n}(2 n z \cos \Phi)=\frac{1}{2} z^{2} \cos ^{2} \Phi$

we find

$\sum_{n=1}^{\infty} n^{-2} J_{n}^{2}(n z)=\frac{1}{4}-\frac{z^{2}}{\pi} \int_{0}^{\pi / 2} \mathrm{~d} \Phi \cos (2 \Phi) \cos ^{2} \Phi$.

It is very easy to solve this integral and we finally find

$\sum_{n=1}^{\infty} n^{-2} J_{n}^{2}(n z)=\frac{1}{4}-\frac{z^{2}}{8}$

if $|z| \leq 1$. 